\title{
The AP2/ERF Transcription Factor SIERF.F5 Functions in Leaf Senescence in Tomato
}

\section{Yanan Chen}

Chongqing University

\section{Panpan Feng}

Chongqing University

\section{Boyan Tang}

Chongqing University

\section{Zongli Hu}

Chongqing University

\section{Qiaoli Xie}

Chongqing University

\section{Shuang Zhou}

Henan University of Science and Technology

Guoping Chen ( $\nabla$ chenguoping@cqu.edu.cn )

Chongqing University

\section{Research Article}

Keywords: SIERF.F5, leaf senescence, ethylene, jasmonate, SIMYC2, tomato

Posted Date: April 12th, 2021

DOI: https://doi.org/10.21203/rs.3.rs-306723/v1

License: (c) (1) This work is licensed under a Creative Commons Attribution 4.0 International License.

Read Full License

Version of Record: A version of this preprint was published at Plant Cell Reports on March 3rd, 2022. See the published version at https://doi.org/10.1007/s00299-022-02846-1. 


\section{Abstract}

The process of plant senescence is complex and highly coordinated, and is regulated by many endogenous and environmental signals. Ethylene and jasmonic acid are well-known senescence inducers, but their molecular mechanisms for inducing leaf senescence have not been fully elucidated. Here, we studied a receptor gene downstream of an ethylene signal transduction pathway, ETHYLENE RESPONSE FACTOR F5 (SIERF.F5). The silence of SIERF.F5 causes accelerated senescence induced by age, darkness, ethylene, and jasmonic acid. However, overexpression of SIERF.F5 may delay leaf senescence. We further found that silencing of SIERF.F5 inhibited the expression of chlorophyll-related genes CHLH, CHLM, POR, CAO1, GUN4, PPH, SGR1, RBCS, and AUREA genes, and the light-responsive RBCS and LHCA1 gene. Moreover, silencing of SIERF.F5 increases the sensitivity of SIERF.F5-RNAi lines to ethylene and jasmonic acid compared to wild type. In the dark-induced aging experiment, the qRT-PCR analysis showed the expression levels of genes related to the ethylene biosynthesis pathway and the jasmonic acid signaling pathway in SIERF.F5-RNAi lines increased compared with wild type. Yeast two-hybrid experiments showed that SIERF.F5 and SIMYC2 (a transcription factor downstream of the JA receptor) can interact physically, thereby mediating the role of SIERF.F5 in jasmonic acid-induced leaf senescence. Collectively, our research provides new insights into how ethylene and jasmonic acid promote leaf senescence in tomatoes.

\section{Key Message}

Our findings not only confirmed that SIERF.F5 can regulate tomato leaf senescence, but also provided new insights into the mechanism of how ethylene and jasmonic acid promote tomato leaf senescence.

\section{Introduction}

Leaf senescence is a necessary process in the growth and development of plants. It's an active and controllable process. During the senescence process, plant leaf cells have undergone tremendous changes in the structure, metabolism, and gene expression in a programmed manner (Lim, Kim and Nam 2007, Zhao et al. 2018). Decomposition of the chloroplast is one of the earliest and most noticeable changes in cell structure. In terms of metabolism, the main differences are the loss of photosynthesis and the hydrolysis of macromolecular substances, such as proteins and nucleic acids (Woo et al. 2013, Lim and Nam 2005). These hydrolyzed molecules are transported to the developing fruits and seeds, which are very important for plant survival and sustainability in annual plants (Woo et al. 2013, Bresson et al. 2018).

Leaf senescence is the last step of plant leaf growth and development, and it is affected by growth, hormones, and external environment, such as age, darkness, drought, and pathogen attacks (Lim et al. 2007). Among the many factors that affect leaf senescence, plant hormones are essential. According to reports, leaf senescence is affected by the main plant hormones. Among them, ethylene, methyl jasmonate (MeJA), abscisic acid (ABA), salicylic acid (SA), and brassinosteroids can promote 
senescence. However, cytokinins (CKs), gibberellin acid (GA), and auxin can inhibit senescence (Gan and Amasino 1997, Jibran, Hunter and Dijkwel 2013). But so far, the potential regulatory mechanism of leaf senescence and the role of hormones have not been fully elucidated.

As we all know, ethylene is the most common and very crucial plant hormone. It participates in many growth and development processes, including cell elongation, seed germination, flowering, fruit maturation, organ senescence, and response to stress (Johnson and Ecker 1998). It has a positive regulatory effect on fruit ripening and organ senescence (Abeles et al. 1988). However, the cause of leaf senescence does not necessarily require the presence of ethylene. Ethylene can only regulate the time of senescence, and treating young leaves with ethylene will not cause senescence (Jing et al. 2002, Jing et al. 2005, Podzimska-Sroka et al. 2015). According to reports, many transcription factors related to ethylene play an essential role in plant senescence. For example, in Arabidopsis dark-induced leaf senescence experiments, NO can regulate EIN2 (a transcription factor for ethylene signaling) to promote senescence (ETHYLENE INSENSITIVE 2) (Niu and Guo 2012). EIN3, a key transcription factor for ethylene signaling, is constitutively overexpressed or temporarily activated to accelerate leaf senescence symptoms (Li et al. 2013). Besides, jasmonic acid (JA) also plays a central role in the senescence of plant leaves induced by darkness. It can influence the expression of various genes to promote senescence (He et al. 2002, Jung et al. 2007). For example, during JA-induced leaf senescence, the level of WRKY57 protein is reduced, and the wrky 57 mutant produces a typical leaf senescence phenotype in Arabidopsis, WRKY57 can interact with JAZ4/8 (Jiang et al. 2014). In JA-induced leaf senescence, Dof2.1 acts as an enhancer. It mainly enhances leaf senescence by promoting SIMYC2 (the helix-loop-helix transcription factor) expression (Zhuo, Sakuraba and Yanagisawa 2020). Also, SIMYC2 regulating the root growth and the defense of pathogen infections regulated by JA, and it plays a positive regulatory role in JA-induced leaf senescence (Song et al. 2017). Moreover, SIMYC2 is activated by jasmonic acid and can inhibit the activity of EIN3 (Zhang et al. 2014). In the research related to ethylene and jasmonic acid, a group of JAZ proteins (JAZ1, JAZ3, and JAZ9) can directly bind to EIN3 and EIL 1 involved in ethylene signaling (Zhu et al. 2011). According to reports, ethylene and jasmonic acid have been found to coordinate (cooperatively or antagonize) plant growth and development and respond to stress (Li and Guo 2007). However, the molecular mechanism of their coordination has been relatively little studied.

Senescence is caused by a complex network of gene regulation (Nam 1997). Many studies have shown that through differential expression methods, through the identification and characterization of senescence-related mutants and hundreds of senescence-related genes (SAGs), there have been many advances in the understanding of leaf senescence at the molecular level (Buchanan-Wollaston et al. 2003, Li et al. 2012). With the in-depth study of leaf senescence, more and more genes of the transcription factor family have been confirmed to be related to senescence. These transcription factor families mainly include WRKY, NAC, MADS, MYB, bZIP and bHLH (Li et al. 2013). Studies have shown that more than 200 transcription factor families are involved in the regulation of leaf senescence, which indicates that leaf senescence is controlled by a complex transcriptional regulatory network (Liu et al. 2011, Li et al. 2012, Li et al. 2013). For instance, darkness promotes the transcription of AtWRKY22, which is involved senescence regulation (Zhou, Jiang and Yu 2011). In promoting leaf senescence, 
ANAC046 regulates Chl catabolism genes and senescence-related genes in Arabidopsis (Oda-Yamamizo et al. 2016).

The AP2/ERF (APETALA2/Ethylene Response Factor) family is one of the largest plant transcription factors, with approximately 140-280 members in various plants. The functions of many genes in this family have been thoroughly studied. It mostly related to planting growth and development, biological, and abiotic stresses, and fruit maturation, mainly by controlling the response to various plant hormones (ethylene, abscisic acid, and jasmonic acid). They can represent the diversity and specificity of ethylene reactions (Li et al. 2018, Liu et al. 2016, Nakano et al. 2006). The ethylene response factor (ERF) family belongs to the AP2/ERF superfamily, characterized by a highly conserved AP2 DNA binding domain consisting of 60-70 amino acid residues (Ohmetakagi and Shinshi 1995, Sakuma et al. 2002). The ERF domain comprises three $\beta$-sheets and an a-helix, where $\beta$-sheets can recognize various cis-acting elements. The a-helix may be involved in the interaction with other transcription factors or DNA. Based on the differences in the amino acid sequence of the ERF domain, ERF proteins are divided into four categories (Tournier et al. 2003). Among them, the class II ethylene-responsive transcription factor (ERF) contains a conserved repressor domain L/FDLNL/F(x)P at the C-terminus, which is called the ERF-related amphiphilic repressor (EAR) motif or CMVIII-1 motif (Nakano et al. 2006). The unique feature of this family is the ability to recognize GCC-box (AGCCGCC) (Ohmetakagi and Shinshi 1990). GCC-Box is the best target for ERFs class proteins, and class II putative repressor ERFs are more flexible than other proteins (Tournier et al. 2003). ERF generally acts as a transcriptional activator, but ERF containing EAR acts as a GCC-mediated transcriptional repressor (Ohta et al. 2001). ERF transcription factors can interact with genes in other hormone signaling pathways. Research proves that ethylene and MeJA can activate the expression of AtERF1 separately or cooperatively, indicating that AtERF1 plays an essential role in the signal transduction pathway of ethylene and jasmonic acid (Lorenzo et al. 2003).

In recent years, studies have shown that AP2/ERF family genes are also related to senescence, SIERF36 overexpression can promote flowering and senescence (Upadhyay et al. 2013). Although AP2/ERF family genes are related to senescence, its research is still sparse and not deep enough. Here, a gene from the AP2 family, SIERF.F5, was isolated from tomato (Solanum lycopersicum, Mill. cv. Ailsa Craig, $\mathrm{AC}^{++}$). In this article, a study of SIERF.F5 gene silencing was conducted to clarify the function of SIERF.F5 in tomatoes. Studies have shown that under normal and dark and hormone-treated conditions, the SIERF.F5RNAi lines showed accelerated leaf senescence. The morphological, biochemical, and molecular comparisons of WT and SIERF.F5-RNAi lines further confirmed that silencing SIERF.F5 can promote senescence. The yeast two-hybrid experiment shows that SIERF.F5 can interact with SIMYC2, downstream of the JA receptor, SIMYC2 positively regulates leaf senescence induced by JA, thereby promoting senescence. In short, SIERF.F5 may promote senescence under the action of ethylene and MeJA, which provides new ideas for studying the role of ethylene and MeJA in leaf senescence.

\section{Materials And Methods}




\subsection{Plant materials and growth conditions}

The wild-type (WT) tomato (Solanum lycopersicon Mill. cv. Ailsa Craig, $\mathrm{AC}^{++}$) and SIERF.F5-RNAi, SIERF.F5-overexpressing transgenic lines were used in this study. Tomato growth conditions: 16-h day $\left(28^{\circ} \mathrm{C}\right) / 8$-h night $\left(18^{\circ} \mathrm{C}\right)$ cycle, greenhouse. To detect the response of SIERF.F5 to plant hormones, 35-dayold WT tomato seedlings were treated with $50 \mu \mathrm{M}$ abscisic acid (ABA), $50 \mu \mathrm{M}$ indole 3-acetic acid (IAA), $50 \mu \mathrm{M}$ Jasmonic acid (MeJA), $100 \mu \mathrm{M}$ gibberellin (GA3), $50 \mu \mathrm{M}$ 1-aminocyclopropane-1-carboxylate (ACC) and distilled water, respectively. Three treatments were performed for each hormone, with three biological replicates. After $0,1,2,4,8,12$, and 24 hours of treatment, the third leaf was collected from the top of the wild tomato seedlings. To examine the specific expression of SIERF.F5 in tomato, various tissue samples of WT tomato were collected. These tissues include: roots (RT), stems (ST), young leaves (YL), mature leaves $(M L)$, senescent leaves $(S L)$, flowers $(F L)$, immature green $(I M G)$, mature green $(M G)$, break $(B), 4$ days after break $(B+4)$, and 7 days after break $(B+7)$. All these samples were immediately wrapped in foil, frozen with liquid nitrogen and stored in a $-80^{\circ} \mathrm{C}$ refrigerator.

\subsection{Construction of SIERF.F5-RNAi and SIERF.F5- overexpression vectors and plant transformation}

To obtain the SIERF.F5-RNAi transgenic lines, the 485 bp fragment of SIERF.F5 was amplified with the primers SIERF.F5-RNAi-F1/R1 (Supplementary Table S1), and ligated into the pBIN19 vector to form the SIERF.F5-RNAi vector, which can produce hairpin SIERF.F5-specific DNA fragments. The vector construction process was performed according to our previous report (Zhou et al. 2019). To construct the SIERF.F5-overexpressing vector, used primers SIERF.F5-full-F2/R2 (Supplementary Table S1) to amplify the full-length SIERF.F5 cDNA. The amplified products were digested with $\mathrm{Xbal} / \mathrm{Sacl}$ and linked to the plant binary vector $\mathrm{pBI} 121$ under the control of the CaMV 35S promoter at Xbal/Sacl restriction sites. The constructed vector was transformed into tomato cv. Ailsa Craig by Agrobacterium tumefaciens LBA4404 strain through the freeze-thaw method. Finally, transgenic lines were selected by kanamycin $(50 \mathrm{mg} / \mathrm{L})$, and confirmed by PCR using NPTII-F/R primers (Supplementary Table S1).

\subsection{Total RNA extraction and Quantitative reverse- transcription-PCR analysis}

Total RNA was extracted from stored samples using Trizol reagent (Invitrogen, Shanghai, China). RNA extraction method based on our previous research (Xie et al. 2014).

Quantitative reverse-transcription-PCR (qRT-PCR) was performed by using a CFX $96^{\text {TM }}$ RealTime System (Bio-Rad, USA). PCR reaction system: $5 \mu \mathrm{L}$ enzyme solution $\left(2 \times\right.$ GoTaq ${ }^{\circledR q R T-P C R ~ M a s t e r ~ M i x, ~ P r o m e g a), ~}$ $3.5 \mu \mathrm{L}$ distilled water, $0.5 \mu \mathrm{L}$ primer pair $(10 \mathrm{mM})$, and $1 \mu \mathrm{L} \mathrm{CDNA}$. PCR reaction program: $95^{\circ} \mathrm{C}$ for 3 minutes, then at $95^{\circ} \mathrm{C}$ for 15 seconds, and Tm (the most suitable temperature) for 45 seconds for 40 cycles. SICAC with relatively stable expression was selected as the internal reference (Nicot et al. 2005), and the expression level of the gene was analyzed using the $2^{-\triangle \triangle C T}$ method. All samples were repeated three times. The primers used in this experiment were listed in Supplementary Table S1. 


\subsection{Determination of leaf senescence induced by age, darkness, ethylene, and MeJA}

For age-dependent leaf senescence, 10-week-age tomato leaves of WT and SIERF.F5-RNAi lines were sampled, and their chlorophyll contents were measured, respectively. For dark-induced senescence experiments, we selected 10-week-age plants, and detached mature leaves of the same location from WT and SIERF.F5-RNAi lines, and place them on the filter paper containing $3 \mathrm{~mL}$ of distilled water at the bottom of a $150 \mathrm{~mm}$ Petri dishes. Place it in a dark environment and keep it at $22^{\circ} \mathrm{C}$. Take samples at $0 \mathrm{~d}$, $5 \mathrm{~d}$, and $7 \mathrm{~d}$, respectively.

In the experiment of hormone and darkness-induced leaf senescence, mature leaves of WT and RNAi tomato seedlings for 10-week-age were collected and placed on a filter paper in a Petri dish with a diameter of $150 \mathrm{~mm}$. Then $3 \mathrm{~mL}$ of distilled water, $100 \mu \mathrm{M} \mathrm{ACC}, 50 \mu \mathrm{M} \mathrm{MeJA}$, or $10 \mu \mathrm{M} \mathrm{AgNO}{ }_{3}$ were added into the dish, respectively, covered, and placed in a dark environment at $22^{\circ} \mathrm{C}$.

\subsection{Measurement of total chlorophyll and carotenoids content}

To detect the age, darkness, and hormone treatment of the leaf senescence of SIERF.F5-RNAi and WT lines, the contents of chlorophyll and carotenoid were detected. Weigh the fresh leaves, grind them thoroughly with liquid nitrogen, and extract with $80 \%$ acetone. The specific experimental process and calculation method are described by Wellburn et al. (Wellburn 1994). Each experiment was repeated three times in biology, and each determination technique was repeated three times.

\subsection{Measuring malondialdehyde (MDA) and electrolytic leakage}

To detect the malondialdehyde (MDA) content, the fresh leaves were thoroughly ground with liquid nitrogen, $0.2 \mathrm{~g}$ was weighed into a centrifuge tube, and $4 \mathrm{~mL}$ of $10 \%$ trichloroacetic acid (TCA) was quickly added, mixed, and centrifuged at $15,000 \mathrm{~g}$ for 5 minutes. Pipet $1 \mathrm{~mL}$ of the supernatant into a new centrifuge tube, and then add $4 \mathrm{~mL}$ of $10 \%$ trichloroacetic acid solution containing $0.5 \%$ thiobarbituric acid (TBA). After mixing, incubate at $95^{\circ} \mathrm{C}$ for half an hour, then place it in an ice-water mixture to stop the reaction. After 10,000 rpm, $10 \mathrm{~min}$, and measure the absorbance of the supernatant at $532 \mathrm{~nm}$ and 600 $\mathrm{nm}$. Repeat three times for each sample. This method is described by Sanjaya et al. (Sanjaya et al. 2008) and Zhang et al. (Zhang et al. 2009).

For the detection of electrical conductivity, take tomato leaves of comparable size (try to ensure the integrity of the leaves, with few stems and nodules), rinse with tap water and rinse with distilled water three times, absorb the surface moisture with filter paper, and take a punching method, avoiding the main vein, each 20 round leaves were placed in a graduated test tube of $50 \mathrm{~mL}$, added with $20 \mathrm{~mL}$ of distilled water, and soaked at $28^{\circ} \mathrm{C}$ for $12 \mathrm{~h}$. Measure the conductivity of the leaching solution (R1) with a conductivity meter, then place the test tube in boiling water for 30 minutes, and cool to $28^{\circ} \mathrm{C}$, shake it up, 
and measure the conductivity of the leaching solution again (R2), relative electrical conductivity $=R 1 / R$ 2*100\%.

\subsection{Superoxide dismutase (SOD)}

For the determination of superoxide dismutase (SOD) activity, the WST method was used, and the operation steps refer to the WST method kit instructions.

\subsection{Yeast two-hybrid}

The open reading frame of SIERF.F5 was amplified by PCR using primers SIERF.F5-F and SIERF.F5-R (Table 1S). The PCR product was digested with Smal and BamHI, and cloned into the pGBKT7 bait vector to obtain the vector SIERF.F5-pGBKT7. At the same time, using the primer pairs SIMYC2-F and SIMYC2-R, the open reading frames of SIMYC2 were amplified by PCR (Table 1S), and digested with Smal and $B a m H I$, cloned into the pGADT7 vector to obtain the vectors SIMYC2-pGADT7. Then the constructed vectors were transferred into Y2Hgold, respectively. Yeast two-hybrid with bait was plated on SD medium lacking Trp, Leu, and SD medium lacking Trp, His, Ade, Leu to test the self-activation of SIERF.F5-pGBKT7, SIMYC2-pGADT7. SIERF.F5-pGBKT7 and SIMYC2-pGADT7 were co-transformed into Y2Hgold. Plated it on SD medium lacking Trp, Leu, and culture it upside down for three days. Pick single colonies on SD medium lacking Trp, His, Ade, Leu, and culture it upside down for 1-2 days, and use X-a-Gal (QDO/X) to judge whether SIERF.F5 can interact with SIMYC2.

\subsection{Statistical analysis}

Data were presented as mean \pm standard deviation. A significant difference between transgenic lines and WT were analyzed using the Student's t-test $(*, P<0.05)$.

\section{Results}

\subsection{Sequence and phylogenetic tree analyses of SIERF.F5}

Based on the sequence in the Tomato Genome Database (https://solgenomics.net, accession No. Solyc10g009110), SIERF.F5 contains 1466 base pairs (bp) encoding a putative protein of 222 amino acids. SIERF.F5 is named by Liu (Liu et al. 2016). Multi-sequence alignment of proteins based on DNAMAN. This protein contains a typical AP2-domain. AP2 domains are typically characterized by three $\beta$-sheets and one a-helix (Fig. 1a). Based on previous studies, SIERF.F5 belongs to the class II putative repressor ERFs.

The phylogenic tree was calculated by MEGA (Molecular Evolutionary Genetics Analysis) version 5.0. Phylogenetic analysis based on selected full-length protein sequences of ERF showed that SIERF.F5 is most related to NtEREBP5, followed by AtERF4, AtERF3, SodERF3 (Fig. 1b). Currently, there is no research on the NtEREBP5 gene. Regarding AtERF4, it acts as a class II repressor and can be induced by ethylene, jasmonic acid, and abscisic acid (Yang et al. 2005). Besides, in the case of iron deficiency, AtERF4 can regulate the expression of these two genes by combining the promoters of AtCLH1 and AtITR1, thereby 
playing a negative regulatory role in the stress process (Liu et al. 2017a). SodERF3 belongs to class II putative repressor ERF and can be bind to GCC-box. Overexpression of SodERF3 improves tolerance to drought and salt in tobacco (Trujillo et al. 2008). In the previous research of SIERF.F5, it is mainly related to stress, but its role in tomato growth and development has not been reported.

\subsection{Expression pattern analysis of SIERF.F5}

To clarify the role of SIERF.F5 in tomato growth and development, the accumulation of its transcripts in various tissues is quantified by qRT-PCR. Figure 2 a shows that the expression level of SIERF.F5 in leaves, $M G$, and $\mathrm{B}+4$ fruit is relatively high, while it is low in other tissues. The specific expression of SIERF.F5 suggests that it may play a role in leaves and fruits. We sought to study further the role of SIERF.F5 in leaf growth and development. We examined young tomato leaves $(Y)$, mature leaves $(M)$, early senescent leaves (leaf yellowing area $>25 \%, E L$ ) and late senescent leaves (leaf yellowing area $>50 \%$, LS) (Fig. 2b). The mRNA level detected by qRT-PCR confirmed that the transcription level of SIERF.F5 gradually decreases during leaf development and senescence (Fig. 2c). We also test two photosynthesis genes Cab7 (chlorophyll/binding protein 1), RBCS (ribose bisphosphate carboxylase small chain), and RAV1 (related to $A B I 3 / V P 1$ ), and their expression levels gradually decreased as the leave senescence (Fig. 2d-f). As a positive control for senescence, SISAG12 is a widely used molecular marker for leaf senescence, expressed explicitly in senescent leaves (Fig. 1g). Here, only to show the relative expression of genes, so the expression level of WT is set to one in the qRT-PCR results. The expression level of SIERF.F5 gradually decreases with leaf senescence, which indicates that silencing the expression level of SIERF.F5 might affect leaf senescence.

Given that SIERF.F5 is a downstream receptor component of the ethylene signal transduction pathway. It is closely related to ethylene, to further confirm whether SIERF.F5 is affected by other hormones, we used qRT-PCR to examine the expression patterns of SIERF.F5 under different hormone treatments. Figure $2 \mathrm{~h}$ shows that the accumulation of SIERF.F5 transcripts increases rapidly to the maximum after one hour of hormone treatment (such as IAA, ABA, MeJA, GA3, and ACC). With the increase of treatment time, the expression level of SIERF.F5 gradually decreases, indicating that hormone treatment may affect the expression of SIERF.F5 in a short time.

\subsection{Silencing of SIERF.F5 accelerates the senescence of tomato leaves}

To further clarify the effect of SIERF.F5 on tomato growth and development and the role of other phytohormones, we constructed the SIERF.F5-RNAi vector and obtained the transgenic lines through the Agrobacterium-mediated genetic transformation method. Examination of its silencing efficiency (Fig. 3a) shows that compared with the WT, the expression of SIERF.F5 in the leaves of the five transgenic lines was significantly reduced by $94-98 \%$. The best silencing efficiency lines are 5,13 , and 15 . Because the 5 and 15 lines harvested few seeds, they could not support the next research, so we chose the 10,13, and 16 lines, called RNAi10, RNAi13, and RNAi16, and conduct further research. At the age of 6 weeks of tomato seedlings, the silent lines show premature senescence (Fig. 3b), and Fig. $3 c$ shows the leaves of 
the same part of the WT and SIERF.F5-RNAi lines. The leaves of RNAi lines appear yellow, while the leaves of WT are still greener. To see the color and shape of each leaf more clearly, the leaf is split and taken a photo (Fig. 3d). Because of the senescent phenotype of the leaves, we test their chlorophyll content. The 6-week-old tomato plants were labeled as the first leaf, the second leaf, and so on, until the sixth leaf. Detect the chlorophyll content of each leaf. From Fig. 3e, we can find that the chlorophyll content of WT leaves is slightly higher than that of RNAi leaves at different leaf ages. Further statistics on the senescence time of tomato leaves reveal that the senescence time of RNAi lines is earlier than that of WT (Fig. 3f). Compare with the wild type, the leaf senescence time of RNAi lines is advanced by about one week. These data demonstrated that silencing of SIERF.F5 could promote the senescence of tomato leaves.

\subsection{Silencing of SIERF.F5 promotes dark-induced leaf senescence}

Dark-induced leaf senescence is a common way to study senescence (Li et al. 2013). To further study the role of SIERF.F5-RNAi in leaf senescence, we take mature leaves for in vitro experiments. As shown in Fig. 4a, the edge of leaves of the SIERF.F5-RNAi lines start to become yellow when treating for five days in the dark, and at seven days, some leaves turned almost completely yellow, and the edges of the remaining leaves had turned yellow, whereas, the leaves of the WT are still green after seven days treatment. To further confirm the phenotype of senescence, we measure some physiological indicators related to leaf senescence. First, detecting the chlorophyll content, we find that at day 0 , the chlorophyll content of the RNAi lines is higher than that of the WT. This may be because the leaves of the RNAi lines are more mature, and the nutritional status was better than that of the WT. However, with the dark treatment time increase, the chlorophyll content of SIERF.F5-RNAi lines leaves gradually decrease. It is significantly lower than that of WT at $7 \mathrm{~d}$, while the chlorophyll content of WT leaves only decreased slightly during the treatment (Fig. 4b). MDA is an important indicator of membrane damage (Sanjaya et al. 2008). Compared with WT, the MDA content in the leaves of the SIERF.F5-RNAi lines is significantly higher. When treating in the dark for $5 d$ to $7 d$, the rate of increase of MDA content in the leaves of RNAi lines is slightly higher than that of WT (Fig. 4c). Further, the electrical conductivity is also an indicator of the membrane damage of the blade. At $0 \mathrm{~d}$, the electrical conductivity of the WT blade is slightly higher than that of the SIERF.F5-RNAi blade. This may be due to the damage received by sampling, but at $7 \mathrm{~d}$, the electrical conductivity of RNAi lines leaves is higher than that of WT leaves (Fig. 4d). Besides, the activity of superoxide dismutase (SOD) is also an indicator of the degree of damage to the cell membrane. At $0 \mathrm{~d}$, the SOD activity of the RNAi lines' leaves is lower than that of WT (Fig. 4e), which may be caused by damage to the leaves. however, Fig. 4e shows that after five days, compared with WT, the SOD activity of SIERF.F5-RNAi leaves is significantly lower. These results indicate that silencing of SIERF.F5 gene leads to premature leaf senescence under dark conditions.

\subsection{Silencing of SIERF.F5 affects the expression of chlorophyll, ethylene, and jasmonic acid related genes}


To reveal the possible molecular mechanism in WT and SIERF.F5-RNAi dark-treated senescent leaves for five days, qRT-PCR is used to detect some genes related to chlorophyll. The transcription levels of chlorophyll genes, including magnesium chelatase $\mathrm{H}$ subunit $(\mathrm{CH} L H), \mathrm{Mg}$ protoporphyrin IX methyltransferase $(C H L M)$, protochlorophyllide reductase $(P O R)$, and chlorophyllide an oxygenase (CAO1), pheophytin pheophorbide hydrolase (PPH), STAY-GREEN Protein (SGR1), AUREA and the genomes uncoupled 4 (GUN4), are significantly down-regulated in SIERF.F5-RNAi transgenic lines (Fig. 5ah). Among the genes, SGR1 can induce chlorophyll degradation, which is down-regulated in RNAi lines. However, the chlorophyll content of the leaves of RNAi lines is lower than that of WT, indicating that the decline of chlorophyll content is regulated by a network of many genes. The down-regulation of a single SGR1 gene does not affect the change of chlorophyll content. Besides, the expression level of ribulose bisphosphate carboxylase small chain (RBCS) and light-harvesting protein complex 1 (LHCA1) is also down-regulated in the SIERF.F5-RNAi lines (Fig. 5i, j). A molecular marker widely used for leaf senescence, $S I S A G 12$, can be used as a positive control. Figure $5 \mathrm{k}$ shows that $S I S A G 12$ is significantly upregulated in the SIERF.F5-RNAi lines compared to WT. According to these results, we speculate that silencing of SIERF.F5 gene affects the expression of some genes in the pathway of chlorophyll, thereby reducing the chlorophyll content, which is one of the reasons for leaf senescence.

To investigate the ethylene's role in leaf senescence of the silenced SIERF.F5lines, some of the ethylene signal synthesis pathway genes are examined. Expression of 1-aminocyclopropane-1-carboxylate oxidase 1 (ACO1), 1-aminocyclopropane-1-carboxylate synthase 2 (ACS2), 1-aminocyclopropane-1carboxylate synthase 4 (ACS4) are up-regulated in the SIERF.F5-RNAi lines compared to WT (Fig. 6a-c), indicating that silencing of SIERF.F5 may increase the ethylene content, thereby promoting leaf senescence. Since MeJA plays a positive regulatory role in leaf senescence, and recent years, studies have pointed out that SIMYC2 can regulate plant growth and development through physical interaction with EIN3. Thus, the expression levels of MeJA signaling pathway transcription factors are detected. JASMONATE ZIM-domain (JAZ) genes (in. JAZ1, JAZ2, JAZ4, JAZ7, JAZ11) are up-regulated in the SIERF.F5-RNAi lines (Fig. 6d-h). Besides, the expression of SIMYC2, a transcription factor downstream of the JA receptor, in the leaves of RNAi lines is higher than that of WT (Fig. 6i). CO/1 is an essential regulator of JA-induced leaf senescence. Figure $6 \mathrm{~g}$ shows that its expression in the leaves of SIERF.F5RNAi lines is also higher than that of WT. The above experimental results indicated that suppression of SIERF.F5 gene could increase the gene expression of ethylene biosynthesis, jasmonic acid signal transduction, and receptor downstream transcription factors, which may increase the content of ethylene and jasmonic acid and cause leaf senescence.

\subsection{Ethylene and jasmonic acid accelerate the senescence of SIERF.F5-RNAi leaves in dark conditions}

To further clarify the role of ethylene and jasmonic acid in promoting leaf senescence, we did a triple reaction induced by ACC and a sensitivity test of MeJA. $0 \mu \mathrm{M}, 5 \mu \mathrm{M}, 10 \mu \mathrm{M}$ ACC (1-aminocyclopropane-1carboxylic acid) is used to treat the germinated seeds of WT and SIERF.F5-RNAi lines. After five days of 
cultivation in the dark, SIERF.F5-RNAi lines show a slightly lower length of hypocotyls and root, the lighter weight of seedling than the WT (Supplementary Fig. S1a-d), suggesting that silenced-SIERF.F5 seedling is more sensitive to ACC. Besides, we have noticed that at $0 \mu \mathrm{M}$, compared with WT, the seedling root length of the RNAi lines is shorter, and the seedling weight is heavier, indicating that under normal circumstances, the seedling growth of the RNAi lines is better than that of WT. To further confirm this phenotype, the expression levels of $A C O 1$ and ACS2 in seedling hypocotyls are analyzed by qRT-PCR, and results show that after treatment with ACC $(10 \mu \mathrm{M})$, the transcripts of the two genes in silenced-SIERF.F5 seedlings are higher than those in WT (Supplementary Fig. S1e,f). In the MeJA sensitivity experiment, After seven days of culture under normal conditions, the length of the hypocotyl, root, and seedling weight of the RNAi lines are lower than those of the WT (Supplementary Fig. S2a-d). These results indicate that silenced SIERF.F5 lines are more sensitive to MeJA.

To verify the role of ethylene and jasmonic acid in leaf senescence, we use detached leaves to carry out hormone-induced senescence experiments. We added hormone treatment (ACC and MeJA) in the darkinduced experiment. AgNO3 is an inhibitor of ethylene action in plants. The purpose of treatment with AgNO3 and MeJA is to test the effect of jasmonic acid on leaf senescence in the absence of ethylene. The results show that after seven days of hormone treatment in dark conditions, the leaves of the SIERF.F5-RNAi lines are yellower than WT (Fig. 7a). Compared with WT, the chlorophyll content of SIERF.F5-RNAi is significantly lower (Fig. 7b), and the MDA content is higher (Fig. 7c). The results demonstrate that both ethylene and jasmonic acid treatments can promote leaf senescence of SIERF.F5RNAi lines under dark conditions. Also, jasmonic acid alone can also promote leaf senescence of SIERF.F5-RNAi lines, indicating that ethylene and jasmonic acid synergistically promote leaf senescence.

\subsection{Overexpression of SIERF.F5 may delay dark-induced leaf senescence}

To further verify the function of SIERF.F5 on the leave senescence, we also constructed a SIERF.F5 overexpression vector, transformed it into tomato cotyledon, and obtained transgenic lines overexpressing SIERF.F5. qRT-PCR is used to detect the expression level of SIERF.F5. As shown in Fig. 8a, we selected lines 1, 5, and 6 (OE1,OE5, and OE6) with higher expression efficiency for the next experiment. Similarly, a dark-induced leaf senescence experiment is conducted. The leaves of 10-week-old seedlings of WT, SIERF.F5-RNAi, and SIERF.F5-OE lines are harvest, respectively, and the dark-induced senescence experiment is carried out in the same way as above. After five days, the leaves of SIERF.F5-RNAi plants began to turn yellow, while no noticeable color change occurred in the leaves of WT and SIERF.F5-OE lines (Fig. 8b). Compared with WT, the chlorophyll and carotenoid content of SIERF.F5-OE lines are slightly higher (Fig. 8c and 8d). The chlorophyll content of RNAi and overexpressing lines and WT for five days after dark treatment are sorted together for comparison (Fig. 8e). The results showed that the chlorophyll content of the leaves of the SIERF.F5-OE lines is slightly higher than that of the WT after dark treatment for five days, but at five days the greenness of WT leaves remained more than that of SIERF.F5-RNAi lines, and the total chlorophyll content is higher. Also, after five days the SOD activity of RNAi leaves is lower

Page $11 / 30$ 
than that of WT, whereas the SIERF.F5-OE lines leaves show higher SOD activity than that of WT (Fig. 8f). The above results suggest that suppression of SIERF.F5 can promote leaf senescence, and overexpression of SIERF.F5 might inhibit leaf senescence.

\subsection{SIERF.F5 interacts with SIMYC2}

Based on the above research results, we have obtained that SIERF.F5-RNAi lines displayed promoted leaf senescence under normal growth conditions and under the treatment of dark, ethylene, and jasmonic acid. Given that SIMYC2 is a vital transcription factor downstream of the jasmonic acid receptor and it has a direct relationship with aging, its interaction with SIERF.F5 is the first choice for studying the regulation of aging by ethylene and jasmonic acid. Therefore, we selected SIMYC2 protein to perform

yeast two-hybrid experiments. Results showed that the yeast cells co-expressing SIERF.F5-BD and SIMYC2-AD could grow on the quadruple dropout medium (SD/-Leu-Trp-His-Ade), the same as the yeast cells carrying pGADT7-T and pGBKT7-53 (Positive control), indicating that there is a physical interaction between SIERF.F5 and SIMYC2 (Fig. 9). The results show that SIERF.F5 can interact with SIMYC2 and play an essential role in leaf senescence induced by ethylene and jasmonic acid.

\section{Discussion}

\section{Discussion}

SIERF.F5 belongs to the AP2/ERF family, studies of this family gene major in plant growth, biological and abiotic stress, and fruit ripening. In Arabidopsis, AtERF4 and AtERF8 belong to class II ERF. LeERF3b accumulates before the fruit matures and then declines sharply, and drought and low temperature can significantly induce the expression of LeERF3b (Chen, Hu and Grierson 2008). Overexpression of SIERF.B3-SRDX leads to a significant delay in fruit ripening time, increased fruit softening, and reduced pigment accumulation (Liu et al. 2014). Overexpression of SIERF5, as a class III ERFs protein, can increase tolerance to drought and salt (Pan et al. 2012). In Arabidopsis thaliana, ethylene response factor 53 (AtERF53) affects the expression of drought-responsive genes by combining GCC-box and, or dehydration response elements in the downstream gene promoter, and AtERF53 plays a negative regulatory role in drought stress by affecting the transcriptional activity of RGLG2 (Cheng et al. 2012). Under iron deficiency conditions, AtERF72 can affect the expression of chlorophyll-degrading genes pheophytin an oxidase (PAO) and chlorophyllase (CLH1), and ERF72 can directly bind to the promoter regions of $I R T 1, H A 2$, and $C L H 1$, thereby regulating the plant's lack response to iron stress (Liu et al. 2017b). TERF2/ LeERF2 plays a positive regulatory role in ethylene biosynthesis, and it can enhance the freezing resistance of plants (Zhang and Huang 2010). JERF3 can activate the expression of DRE and CE1 genes containing GCC-box, thereby reducing the accumulation of ROS and enhancing the adaptability of tobacco to drought, low temperature, and high salt (Wu et al. 2008). According to the function of family genes and previous studies, SIERF.F5 may also affect plant growth, stress, and fruit ripening. 
In this study, along with the growth and development of leaves, the expression level of SIERF.F5 gene gradually decreases (Fig. 2c). The expression pattern of SIERF.F5 gene was similar to Cab7, RBCS, RAV1, but it was opposite to the expression pattern of SISAG12. Whether it was age-dependent senescence (Fig. 3b), darkness-induced senescence (Fig. 4a), or hormone-induced senescence (Fig. 7a), the leaves of the SIERF.F5-RNAi lines showed earlier senescence than WT. These experimental results indicated that SIERF.F5 plays a negative role in leaf senescence. Besides, we also created the SIERF.F5-OE tomato lines. In the dark-induced senescence experiment, compared with WT plants, the chlorophyll content, SOD activity, and carotenoid content of the SIERF.F5-overexpression lines were slightly higher, and no apparent yellowing phenomenon was observed (Fig. 8b-f). This indicated that overexpression of SIERF.F5 would not promote senescence. On the contrary, senescence may be delayed. Chlorophyll content reflects the senescence of the leaves. Similarly, compared with WT plants, we found that the chlorophyll content of SIERF.F5-RNAi lines at $5 \mathrm{~d}$ and $7 \mathrm{~d}$ were significantly lower (Fig. 4b). Next, we measured the expression levels of some genes for chlorophyll (CHLH, CHLM, POR, CAO1, GUN4, PPH, SGR1, AUREA), and lightresponsive gene (RBCS, $L H C A 1)$. In researches related to chlorophyll biosynthesis and degradation genes, compared with WT plants, PPH, PAO, RCCR, SGR1 in the SIOFP20-OE line was significantly increased. Overexpression of SIOFP20 can regulate chlorophyll accumulation and leaf senescence (Zhou et al. 2019). The expression levels of RBCS1A and $C A B 1$ were examined to investigate the relationship between EIN3 and leaf senescence (Li et al. 2013). During leaf senescence, the expression of SINAP2 increased. SINAP2 can activate the expression of SISGR1 and SIPAO to regulate senescence (Ma et al. 2018). According to previous research, the expression changes of chlorophyll synthesis and metabolism genes were mostly related to leaf senescence. In this article, these genes were down-regulated in the senescent leaves of the SIERF.F5-RNAi lines, which clarified the mechanism of leaf senescence from a physiological and molecular level.

Among the main hormones that affect leaf senescence, ethylene, as promoters of leaf senescence, play a signicant role in age and darkness-induced senescence. During darkness-induced leaf senescence, ethylene-insensitive mutants (ein2/ ore3) act as senescence promoting factors through transcriptional regulation of stress-related responses (Kim et al. 2018). In the ein3 eil1 double mutant, ethylene inhibits the expression of NYE1, NYC1, and PAO containing GCC-box, and EMSA results indicate that EIN3 can directly bind $N Y E 1, N Y C 1$, and $P A O$ promoters and play central a role in ethylene-mediated leaf senescence (Qiu et al. 2015). Therefore, we supposed that ethylene might be responsible for the leaf senescence of SIERF.F5-RNAi lines. In this study, the expression levels of ACO1, ACS2, ACS4, the ethylene biosynthetic pathway genes in the SIERF.F5-RNAi lines were significantly increased compared with WT, suggesting that suppression of SIERF.F5 might induce the biosynthesis of ethylene, thereby promoting senescence.

Jasmonic acid is also a promoting factor of leaf senescence, and changes in the expression of jasmonic acid-related genes are critically related to leaf senescence. In research on leaf senescence, the expression of $J A Z 7$ was up-regulated in darkness-induced senescence. The jaz7 mutant showed a large area of yellowing of the leaves. In addition, the double mutants of jaz7 SIMYC2 and jaz7 coi1 showed delayed leaf senescence. In conclusion, JAZ7 protein as a regulator of dark-induced leaf senescence (Yu et al. 
2016). JAZ4 and JAZ8 can physically interact with WRKY57 and play a negative regulatory role in MeJAinduced leaf senescence (Jiang et al. 2014). In MeJA-induced senescence experiments, MYC5 overexpressing transgenic plants showed early leaf senescence phenotypes, including reduced chlorophyll content. It enhanced JA-regulated senescence-related gene expression (SAG13, SEN4, SAG113, and SAG29) and photosynthesis genes (RBCS and CAB1) (Song et al. 2017). In the mechanism of MeJA-induced leaf senescence, COI1-dependent JA inhibition was considered to be very important (Shan et al. 2011). The experimental results showed that the expression levels of JAZ1, JAZ2, JAZ4, $J A Z 7, J A Z 11$ in the jasmonic acid signaling pathway in the SIERF.F5-RNAi lines were significantly increased compared with WT (Fig. 6). Besides, the expression of downstream transcription factors of JA receptor (SIMYC2) and COI1 gene was also increased compared with WT (Fig. 6). According to previous reports, increased expression of these genes could promote leaf senescence, may also improve the jasmonic acid content, which may also be one of the reasons for the early senescence of the leaves of the SIERF.F5-RNAi lines.

In the hormone-induced senescence experiment, exogenous addition of ACC and MeJA can induce senescence of SIERF.F5-RNAi leaves. Moreover, the exogenous addition of $\mathrm{AgNO}_{3}$ (an ethylene inhibitor) and MeJA can also induce senescence of SIERF.F5-RNAi leaves. This result indicated that MeJA could also induce senescence in the absence of ethylene (Fig. 7a-c). According to the above results, we can speculate that ethylene and jasmonic acid play a synergistic role in the process of leaf senescence. In the hormone sensitivity experiment, the tomato seedlings of SIERF.F5-RNAi lines showed more sensitive characteristics to ACC and MeJA (Supplementary Fig. S1,2). In summary, suppression of SIERF.F5 gene can promote the leaf senescence by affecting the expression levels of genes in the ethylene biosynthesis pathway, the jasmonic acid signal transduction pathway, and downstream transcription factors of the JA receptor.

According to previous reports, AtERF2 positively regulates jasmonic acid-sensitive defense gene expression, while AtERF4 negatively regulates jasmine-sensitive defense gene expression (McGrath et al. 2005). Given the role of ERF and jasmonic acid-related genes, we choose SIMYC2 and SIERF.F5 for yeast two-hybrid experiments. The results showed that SIERF.F5 could interact with SIMYC2. Studies have shown that increased expression of SIMYC2 gene will promote leaf senescence, which indicated that SIMYC2 plays a positive role in leaf senescence. The experimental results proved that SIERF.F5 plays a negative regulatory role in leaf senescence, and its expression gradually decreases during leaf senescence. In the SIERF.F5-RNAi lines, the expression level of SIMYC2 was higher than that of WT. The above results indicated that silencing of SIERF.F5 gene might relieve its inhibition to SIMYC2, thereby promoting leaf senescence.

Combining previous studies and our experimental results, we propose a working model that describes how SIERF.F5 and SIMYC2 regulate leaf senescence under the influence of hormones and environmental stress (Fig. 10). Ethylene transcription factor SIERF.F5 is an essential negative regulator of leaf senescence. The expression of SISAG12 increased in the leaves of the SIERF.F5-RNAi lines, indicating that inhibition of SIERF.F5 could induce the expression of the SISAG12 gene to promote leaf senescence. 
Under the condition of age or darkness, inhibits the expression of SIERF.F5, the expression levels of $C H L H$, CHLM, POR, CAO1, GUN4, PPH, SGR1, RBCS PPH, SGR1, RBCS, ACREA and LHCA1 were reduced, thereby accelerating leaf senescence. On the one hand, it inhibits SIERF.F5 will induce the expression of SIMYC2, and the two interact to promote leaf senescence in silent lines. On the other hand, SIMYC2 could directly activate SGR1 and other chlorophyll catabolic enzyme genes during the leaf senescence induced by JA (Zhu et al. 2015). Besides, SIERF.F5 can induce the expression of some JAZ proteins in the jasmonic acid signal transduction pathway (JAZ1, JAZ2, JAZ4, JAZ7, JAZ11) among which JAZ7 can interact with COI1 and, or SIMYC2 to regulate leaf senescence induced by darkness (Yu et al. 2016). However, the interaction between JAZ7 and WRKY57 to promote leaf senescence was studied in Arabidopsis thaliana, and its function in tomatoes has not yet been reported. If the regulatory mechanism model was to be perfected, further experimental verification was needed. Overall, the physiological and molecular mechanism analysis showed that SIERF.F5 plays a vital role in regulating the leaf senescence induced by darkness. Ethylene and jasmonic acid play a synergistic role in regulating leaf senescence.

\section{Declarations}

\section{Author contribution statement}

G. C, S. Z, and Z. H designed and managed the research work and improved the manuscript. Y. C, P. F, B. T, Q. X performed the experiments. Y. C wrote the manuscript. All authors read and approved the manuscript.

\section{Acknowledgments}

This work was supported by the National Natural Science Foundation of China (nos.31872121, 31801870) and the Fundamental Research Funds for the Central Universities (2020CDJQY-A059), and the Natural Science Foundation of Chongqing of China (csts2019jcyj-msxmX0094).

\section{Conflict of interest}

All authors have read and approved this version of the article, and due care has been taken to ensure the integrity of this work. The authors declare that they have no conflict of interest.

\section{References}

Abeles, F. B., L. J. Dunn, P. Morgens, A. Callahan, R. E. Dinterman \& J. Schmidt (1988) Induction of 33-Kd and 60-Kd Peroxidases during Ethylene-Induced Senescence of Cucumber Cotyledons. Plant Physiology, $87,609-615$.

Bresson, J., S. Bieker, L. Riester, J. Doll \& U. Zentgraf (2018) A guideline for leaf senescence analyses: from quantification to physiological and molecular investigations. J Exp Bot, 69, 769-786. 
Buchanan-Wollaston, V., S. Earl, E. Harrison, E. Mathas, S. Navabpour, T. Page \& D. Pink (2003) The molecular analysis of leaf senescence-a genomics approach. Plant Biotechnol J, 1, 3-22.

Chen, G., Z. Hu \& D. Grierson (2008) Differential regulation of tomato ethylene responsive factor LeERF3b, a putative repressor, and the activator Pti4 in ripening mutants and in response to environmental stresses. J Plant Physiol, 165, 662-70.

Cheng, M. C., E. J. Hsieh, J. H. Chen, H. Y. Chen \& T. P. Lin (2012) Arabidopsis RGLG2, Functioning as a RING E3 Ligase, Interacts with AtERF53 and Negatively Regulates the Plant Drought Stress Response. Plant Physiology, 158, 363-375.

Gan, S. S. \& R. M. Amasino (1997) Making sense of senescence - Molecular genetic regulation and manipulation of leaf senescence. Plant Physiology, 113, 313-319.

He, Y. H., H. Fukushige, D. F. Hildebrand \& S. S. Gan (2002) Evidence supporting a role of jasmonic acid in Arabidopsis leaf senescence. Plant Physiology, 128, 876-884.

Jiang, Y. J., G. Liang, S. Z. Yang \& D. Q. Yu (2014) Arabidopsis WRKY57 Functions as a Node of Convergence for Jasmonic Acid- and Auxin-Mediated Signaling in Jasmonic Acid-Induced Leaf Senescence. Plant Cell, 26, 230-245.

Jibran, R., D. A. Hunter \& P. P. Dijkwel (2013) Hormonal regulation of leaf senescence through integration of developmental and stress signals. Plant Molecular Biology, 82, 547-561.

Jing, H. C., J. H. M. Schippers, J. Hille \& P. P. Dijkwei (2005) Ethylene-induced leaf senescence depends on age-related changes and OLD genes in Arabidopsis. Journal of Experimental Botany, 56, $2915-2923$.

Jing, H. C., M. J. G. Sturre, J. Hille \& P. P. Dijkwel (2002) Arabidopsis onset of leaf death mutants identify a regulatory pathway controlling leaf senescence. Plant Journal, 32, 51-63.

Johnson, P. R. \& J. R. Ecker (1998) The ethylene gas signal transduction pathway: a molecular perspective. Annu Rev Genet, 32, 227-54.

Jung, C., S. H. Lyou, S. Yeu, M. A. Kim, S. Rhee, M. Kim, J. S. Lee, Y. Do Choi \& J. J. Cheong (2007) Microarray-based screening of jasmonate-responsive genes in Arabidopsis thaliana. Plant Cell Reports, $26,1053-1063$.

Kim, J., S. J. Park, I. H. Lee, H. Chu, C. A. Penfold, J. H. Kim, V. Buchanan-Wollaston, H. G. Nam, H. R. Woo \& P. O. Lim (2018) Comparative transcriptome analysis in Arabidopsis ein2/ore3 and ahk3/ore12 mutants during dark-induced leaf senescence. Journal of Experimental Botany, 69, 3023-3036.

Li, H. J. \& H. W. Guo (2007) Molecular basis of the ethylene signaling and response pathway in Arabidopsis. Journal of Plant Growth Regulation, 26, 106-117. 
Li, Z., J. Peng, X. Wen \& H. Guo (2012) Gene network analysis and functional studies of senescenceassociated genes reveal novel regulators of Arabidopsis leaf senescence. J Integr Plant Biol, 54, 526-39.

Li, Z. H., J. Y. Peng, X. Wen \& H. W. Guo (2013) ETHYLENE-INSENSITIVE3 Is a Senescence-Associated Gene That Accelerates Age-Dependent Leaf Senescence by Directly Repressing miR164 Transcription in Arabidopsis. Plant Cell, 25, 3311-3328.

Li, Z. J., Y. S. Tian, J. Xu, X. Y. Fu, J. J. Gao, B. Wang, H. J. Han, L. J. Wang, R. H. Peng \& Q. H. Yao (2018) A tomato ERF transcription factor, SIERF84, confers enhanced tolerance to drought and salt stress but negatively regulates immunity against Pseudomonas syringae pv. tomato DC3000. Plant Physiology and Biochemistry, 132, 683-695.

Lim, P. O., H. J. Kim \& H. G. Nam (2007) Leaf senescence. Annual Review of Plant Biology, 58, 115-136.

Lim, P. O. \& H. G. Nam (2005) The molecular and genetic control of leaf senescence and longevity in Arabidopsis. Current Topics in Developmental Biology, Vol 67, 67, 49-83.

Liu, M., G. Diretto, J. Pirrello, J. P. Roustan, Z. Li, G. Giuliano, F. Regad \& M. Bouzayen (2014) The chimeric repressor version of an Ethylene Response Factor (ERF) family member, SI-ERF.B3, shows contrasting effects on tomato fruit ripening. New Phytol, 203, 206-18.

Liu, M. C., B. L. Gomes, I. Mila, E. Purgatto, L. E. P. Peres, P. Frasse, E. Maza, M. Zouine, J. P. Roustan, M. Bouzayen \& J. Pirrello (2016) Comprehensive Profiling of Ethylene Response Factor Expression Identifies Ripening-Associated ERF Genes and Their Link to Key Regulators of Fruit Ripening in Tomato. Plant Physiology, 170, 1732-1744.

Liu, W., N. J. U. Karemera, T. Wu, Y. F. Yang, X. Z. Zhang, X. F. Xu, Y. Wang \& Z. H. Han (2017a) The ethylene response factor AtERF4 negatively regulates the iron deficiency response in Arabidopsis thaliana. Plos One, 12.

Liu, W., Q. W. Li, Y. Wang, T. Wu, Y. F. Yang, X. Z. Zhang, Z. H. Han \& X. F. Xu (2017b) Ethylene response factor AtERF72 negatively regulates Arabidopsis thaliana response to iron deficiency. Biochemical and Biophysical Research Communications, 491, 862-868.

Liu, X. C., Z. H. Li, Z. Q. Jiang, Y. Zhao, J. Y. Peng, J. P. Jin, H. W. Guo \& J. C. Luo (2011) LSD: a leaf senescence database. Nucleic Acids Research, 39, D1103-D1107.

Lorenzo, O., R. Piqueras, J. J. Sanchez-Serrano \& R. Solano (2003) ETHYLENE RESPONSE FACTOR1 integrates signals from ethylene and jasmonate pathways in plant defense. Plant Cell, 15, 165-178.

Ma, X., Y. Zhang, V. Tureckova, G. P. Xue, A. R. Fernie, B. Mueller-Roeber \& S. Balazadeh (2018) The NAC Transcription Factor SINAP2 Regulates Leaf Senescence and Fruit Yield in Tomato. Plant Physiol, 177, 1286-1302. 
McGrath, K. C., B. Dombrecht, J. M. Manners, P. M. Schenk, C. I. Edgar, D. J. Maclean, W. R. Scheible, M. K. Udvardi \& K. Kazan (2005) Repressor- and activator-type ethylene response factors functioning in jasmonate signaling and disease resistance identified via a genome-wide screen of Arabidopsis transcription factor gene expression. Plant Physiology, 139, 949-959.

Nakano, T., K. Suzuki, T. Fujimura \& H. Shinshi (2006) Genome-wide analysis of the ERF gene family in Arabidopsis and rice. Plant Physiol, 140, 411-32.

Nam, H. G. (1997) The molecular genetic analysis of leaf senescence. Curr Opin Biotechnol, 8, 200-7.

Nicot, N., J. F. Hausman, L. Hoffmann \& D. Evers (2005) Housekeeping gene selection for real-time RT-PCR normalization in potato during biotic and abiotic stress. J Exp Bot, 56, 2907-14.

Niu, Y. H. \& F. Q. Guo (2012) Nitric Oxide Regulates Dark-Induced Leaf Senescence Through EIN2 in Arabidopsis. Journal of Integrative Plant Biology, 54, 516-525.

Oda-Yamamizo, C., N. Mitsuda, S. Sakamoto, D. Ogawa, M. Ohme-Takagi \& A. Ohmiya (2016) The NAC transcription factor ANAC046 is a positive regulator of chlorophyll degradation and senescence in Arabidopsis leaves. Scientific Reports, 6.

Ohmetakagi, M. \& H. Shinshi (1990) Structure and Expression of a Tobacco Beta-1,3-Glucanase Gene. Plant Molecular Biology, 15, 941-946.

-- (1995) Ethylene-Inducible DNA-Binding Proteins That Interact with an Ethylene-Responsive Element. Plant Cell, 7, 173-182.

Ohta, M., K. Matsui, K. Hiratsu, H. Shinshi \& M. Ohme-Takagi (2001) Repression domains of class II ERF transcriptional repressors share an essential motif for active repression. Plant Cell, 13, 1959-1968.

Pan, Y., G. B. Seymour, C. Lu, Z. Hu, X. Chen \& G. Chen (2012) An ethylene response factor (ERF5) promoting adaptation to drought and salt tolerance in tomato. Plant Cell Rep, 31, 349-60.

Podzimska-Sroka, D., C. O'Shea, P. L. Gregersen \& K. Skriver (2015) NAC Transcription Factors in Senescence: From Molecular Structure to Function in Crops. Plants (Basel), 4, 412-48.

Qiu, K., Z. Li, Z. Yang, J. Chen, S. Wu, X. Zhu, S. Gao, J. Gao, G. Ren, B. Kuai \& X. Zhou (2015) EIN3 and ORE1 Accelerate Degreening during Ethylene-Mediated Leaf Senescence by Directly Activating Chlorophyll Catabolic Genes in Arabidopsis. PLoS Genet, 11, e1005399.

Sakuma, Y., Q. Liu, J. G. Dubouzet, H. Abe, K. Shinozaki \& K. Yamaguchi-Shinozaki (2002) DNA-binding specificity of the ERF/AP2 domain of Arabidopsis DREBs, transcription factors involved in dehydrationand cold-inducible gene expression. Biochemical and Biophysical Research Communications, 290, 9981009. 
Sanjaya, P. Y. Hsiao, R. C. Su, S. S. Ko, C. G. Tong, R. Y. Yang \& M. T. Chan (2008) Overexpression of Arabidopsis thaliana tryptophan synthase beta 1 (AtTSB1) in Arabidopsis and tomato confers tolerance to cadmium stress. Plant Cell Environ, 31, 1074-85.

Shan, X., J. Wang, L. Chua, D. Jiang, W. Peng \& D. Xie (2011) The role of Arabidopsis Rubisco activase in jasmonate-induced leaf senescence. Plant Physiol, 155, 751-64.

Song, S. S., H. Huang, J. J. Wang, B. Liu, T. C. Qi \& D. X. Xie (2017) MYC5 is Involved in JasmonateRegulated Plant Growth, Leaf Senescence and Defense Responses. Plant and Cell Physiology, 58, 17521763.

Tournier, B., M. T. Sanchez-Ballesta, B. Jones, E. Pesquet, F. Regad, A. Latche, J. C. Pech \& M. Bouzayen (2003) New members of the tomato ERF family show specific expression pattern and diverse DNAbinding capacity to the GCC box element. Febs Letters, 550, 149-154.

Trujillo, L. E., M. Sotolongo, C. Menendez, M. E. Ochogavia, Y. Coll, I. Hernandez, O. Borras-Hidalgo, B. P. H. J. Thomma, P. Vera \& L. Hernandez (2008) SodERF3, a novel sugarcane ethylene responsive factor (ERF), enhances salt and drought tolerance when overexpressed in tobacco plants. Plant and Cell Physiology, $49,512-525$.

Upadhyay, R. K., D. K. Soni, R. Singh, U. N. Dwivedi, U. V. Pathre, P. Nath \& A. P. Sane (2013) SIERF36, an EAR-motif-containing ERF gene from tomato, alters stomatal density and modulates photosynthesis and growth. Journal of Experimental Botany, 64, 3237-3247.

Wellburn, A. R. (1994) The Spectral Determination of Chlorophyll-a and Chlorophhyll-B, as Well as Total Carotenoids, Using Various Solvents with Spectrophotometers of Different Resolution. Journal of Plant Physiology, 144, 307-313.

Woo, H. R., H. J. Kim, H. G. Nam \& P. O. Lim (2013) Plant leaf senescence and death - regulation by multiple layers of control and implications for aging in general. Journal of Cell Science, 126, 4823-4833.

Wu, L. J., Z. J. Zhang, H. W. Zhang, X. C. Wang \& R. F. Huang (2008) Transcriptional Modulation of Ethylene Response Factor Protein JERF3 in the Oxidative Stress Response Enhances Tolerance of Tobacco Seedlings to Salt, Drought, and Freezing. Plant Physiology, 148, 1953-1963.

Xie, Q., Z. Hu, Z. Zhu, T. Dong, Z. Zhao, B. Cui \& G. Chen (2014) Overexpression of a novel MADS-box gene SIFYFL delays senescence, fruit ripening and abscission in tomato. Sci Rep, 4, 4367.

Yang, Z., L. N. Tian, M. Latoszek-Green, D. Brown \& K. Q. Wu (2005) Arabidopsis ERF4 is a transcriptional repressor capable of modulating ethylene and abscisic acid responses. Plant Molecular Biology, 58, 585596.

Yu, J., Y. Zhang, C. Di, Q. Zhang, K. Zhang, C. Wang, Q. You, H. Yan, S. Y. Dai, J. S. Yuan, W. Xu \& Z. Su (2016) JAZ7 negatively regulates dark-induced leaf senescence in Arabidopsis. J Exp Bot, 67, 751-62. 
Zhang, L., L. H. Tian, J. F. Zhao, Y. Song, C. J. Zhang \& Y. Guo (2009) Identification of an Apoplastic Protein Involved in the Initial Phase of Salt Stress Response in Rice Root by Two-Dimensional Electrophoresis. Plant Physiology, 149, 916-928.

Zhang, X., Z. Zhu, F. An, D. Hao, P. Li, J. Song, C. Yi \& H. Guo (2014) Jasmonate-activated MYC2 represses ETHYLENE INSENSITIVE3 activity to antagonize ethylene-promoted apical hook formation in Arabidopsis. Plant Cell, 26, 1105-17.

Zhang, Z. J. \& R. F. Huang (2010) Enhanced tolerance to freezing in tobacco and tomato overexpressing transcription factor TERF2/LeERF2 is modulated by ethylene biosynthesis. Plant Molecular Biology, 73, 241-249.

Zhao, L., Y. Xia, X. Y. Wu, J. H. M. Schippers \& H. C. Jing (2018) Phenotypic Analysis and Molecular Markers of Leaf Senescence. Methods Mol Biol, 1744, 35-48.

Zhou, S., X. Cheng, F. Li, P. Feng, G. Hu, G. Chen, Q. Xie \& Z. Hu (2019) Overexpression of SIOFP20 in Tomato Affects Plant Growth, Chlorophyll Accumulation, and Leaf Senescence. Front Plant Sci, 10, 1510.

Zhou, X., Y. J. Jiang \& D. Q. Yu (2011) WRKY22 transcription factor mediates dark-induced leaf senescence in Arabidopsis. Molecules and Cells, 31, 303-313.

Zhu, X., J. Chen, Z. Xie, J. Gao, G. Ren, S. Gao, X. Zhou \& B. Kuai (2015) Jasmonic acid promotes degreening via MYC2/3/4- and ANAC019/055/072-mediated regulation of major chlorophyll catabolic genes. Plant J, 84, 597-610.

Zhu, Z. Q., F. Y. An, Y. Feng, P. P. Li, L. Xue, A. Mu, Z. Q. Jiang, J. M. Kim, T. K. To, W. Li, X. Y. Zhang, Q. Yu, Z. Dong, W. Q. Chen, M. Seki, J. M. Zhou \& H. W. Guo (2011) Derepression of ethylene-stabilized transcription factors (EIN3/EIL1) mediates jasmonate and ethylene signaling synergy in Arabidopsis. Proceedings of the National Academy of Sciences of the United States of America, 108, 12539-12544.

Zhuo, M., Y. Sakuraba \& S. Yanagisawa (2020) A Jasmonate-Activated MYC2-Dof2.1-MYC2 Transcriptional Loop Promotes Leaf Senescence in Arabidopsis. Plant Cell, 32, 242-262.

\section{Figures}


(a)

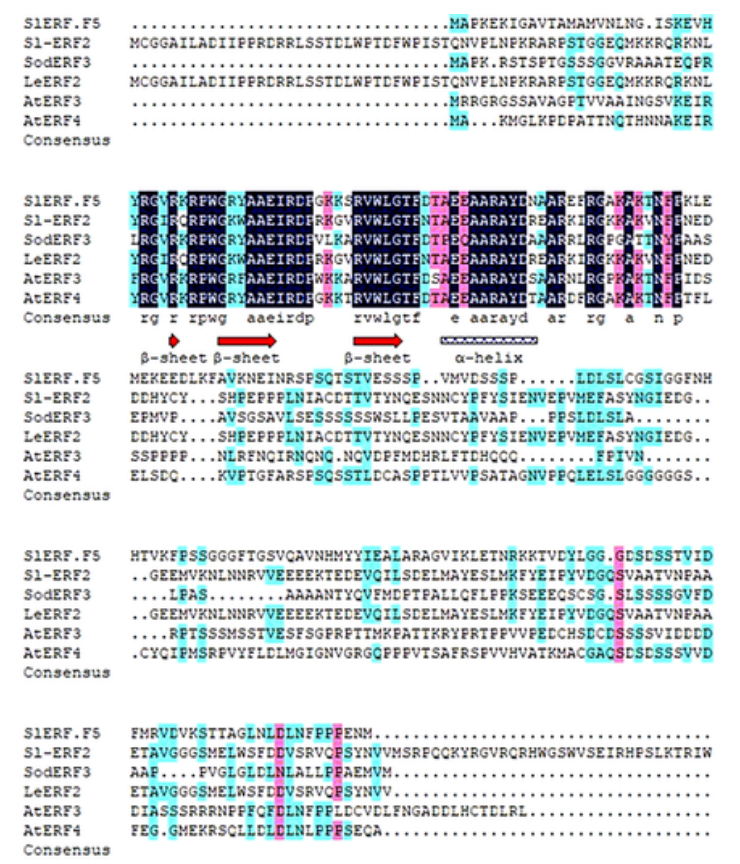

(b)

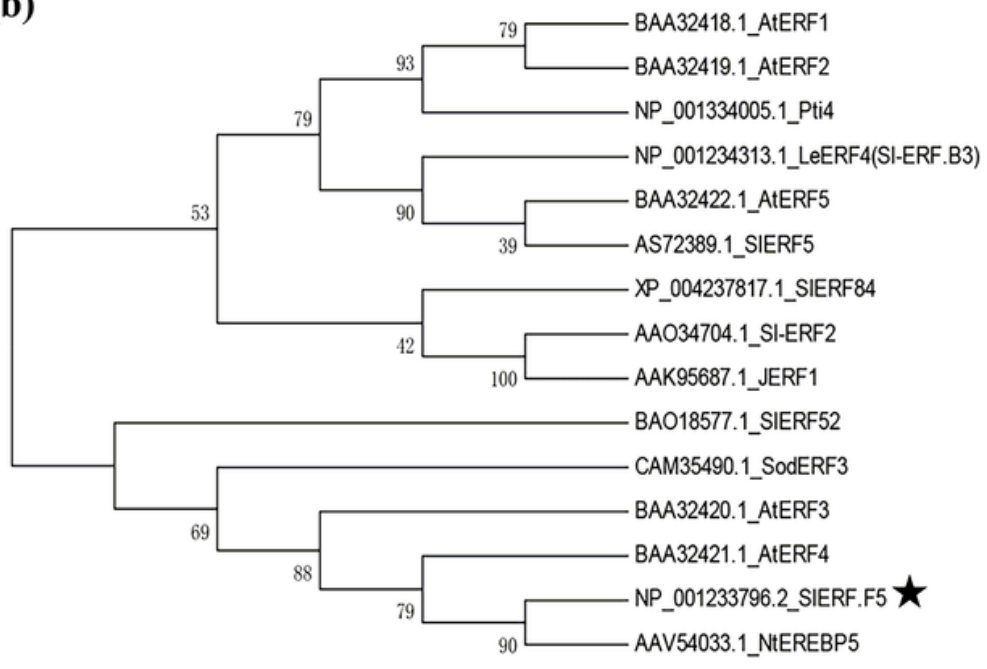

\section{Figure 1}

Sequence and expression analysis of SIERF.F5 (a) Protein sequence alignment of SIERF.F5 and other ethylene transcription factors of ERF family. The same amino acid is indicated in black, and its protein sequence has three $\beta$-sheets and an a-helix. (b) Phylogenetic analysis of SIERF.F5 and other ERF proteins was constructed using MEGA 5.0 software and the Neighbor-Joining method, bootstrap analysis of 1000 replicates. The accession numbers for other proteins are as follows: AtERF1 (BAA32418.1), AtERF2 (BAA32419.1), Pti4 (NP_001334005.1), LeERF4 (SI-ERF.B3) (NP_001234313.1), AtERF5 (BAA32422.1), SIERF5 (AS72389.1), SIERF84 (XP_004237817.1), SI-ERF2 (AA034704.1), JERF1 (AAK95687.1), SodERF3 (CAM35490.1), AtERF3 (BAA32420.1), AtERF4 (BAA32421.1), SIERF.F5 (NP_001233796.2), NtEREBP5 (AAV54033.1), SIERF52 (BA018577.1). هrepresent the genes studied in this article. 
(a)

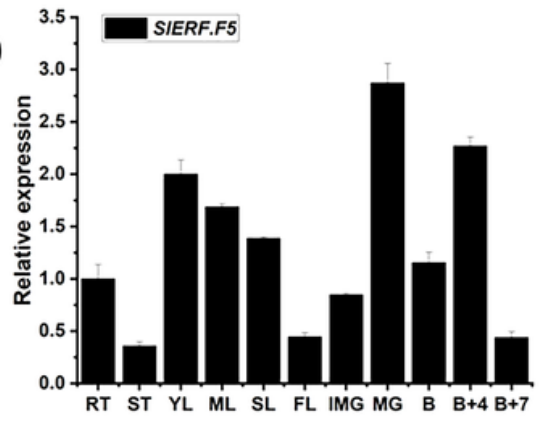

(d)

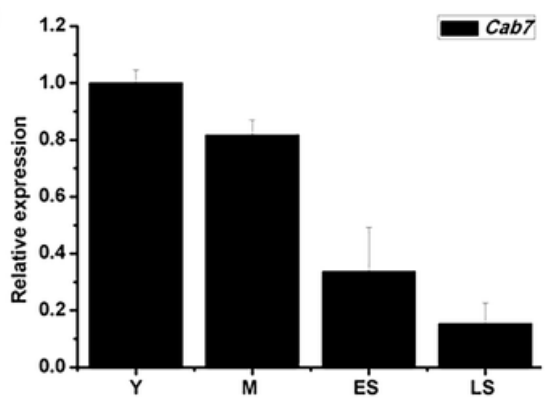

(g)

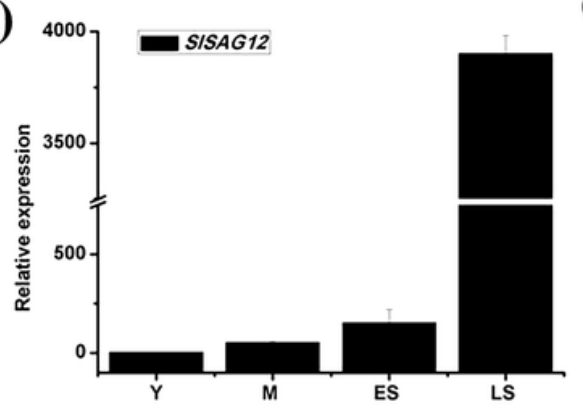

(b)

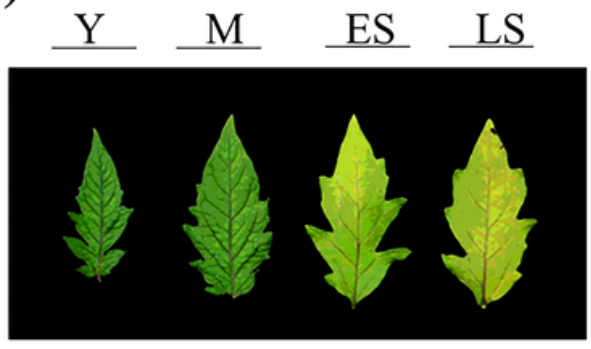

(c)

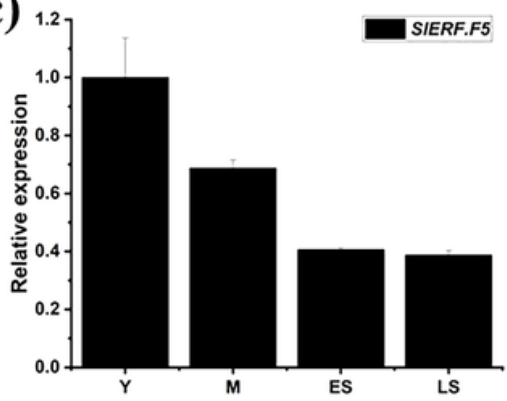

(e)

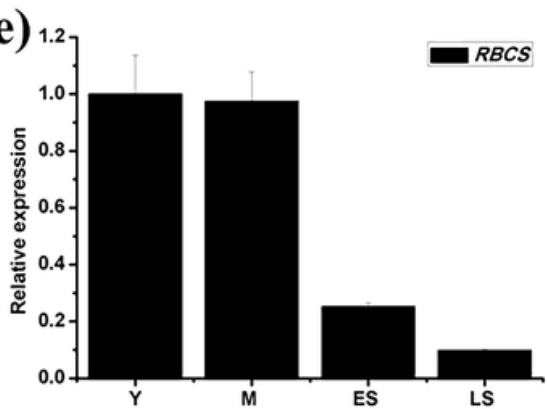

(h)

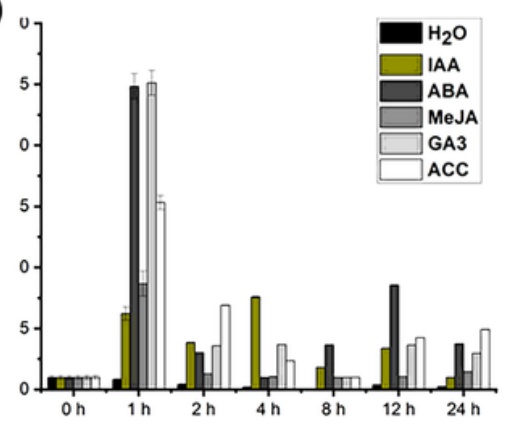

(f)

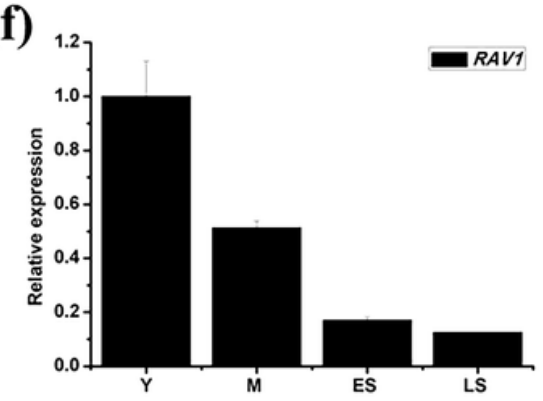

Figure 2

Expression pattern of SIERF.F5 (a) The expression level of SIERF.F5 in various tissues of WT. The expression level of SIERF.F5 in various tissues of WT. RT (root), ST (stem), YL (young leaf), ML (mature leaf), SL (senescence leaf), FL (flower), IMG (immature fruit), MG (green fruit), B (breaker fruit), B+4 (4 days after breaker fruit), $\mathrm{B}+7$ (7 days after breaker fruit). (b) Different development stages of tomato leaves, $Y$ (young tomato leaves), M (mature leaves), EL (early senescence leaves, yellowing area $>25 \%$ ) and LS (late senescence leaves, yellowing area $>50 \%$ ). (c-g) qRT-PCR analysis of the expression levels of SIERF.F5, Cab7, RBCS, Rav7, and SISAG12 in wild-type tomato leaves at different developmental stages. The experimental results were repeated three times in biology. (h) qRT-PCR analysis of the expression patterns of SIERF.F5 in response to IAA, ABA, MeJA, GA3, and ACC. All experimental data are the mean \pm $\mathrm{SD}$ of three independent experiments. Error bars indicate SE. 


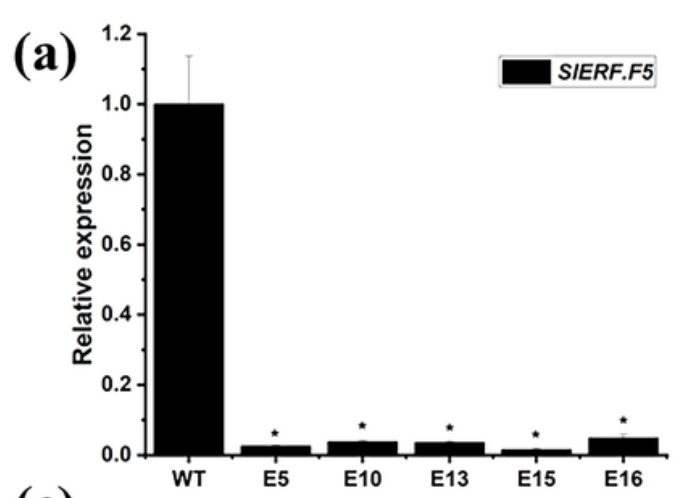

(c)

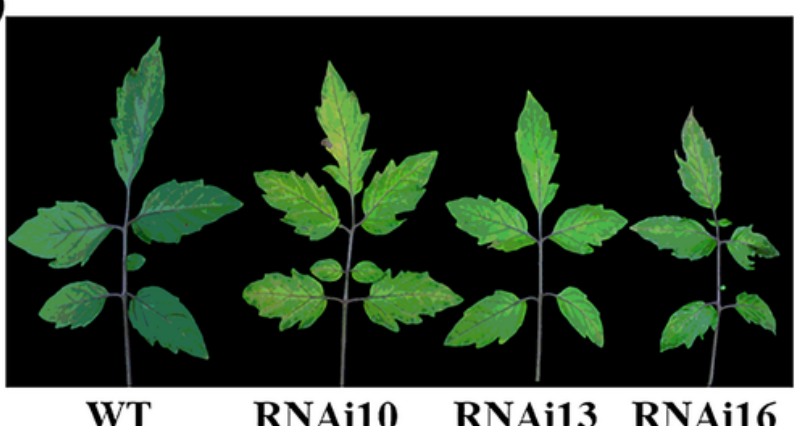

(e)

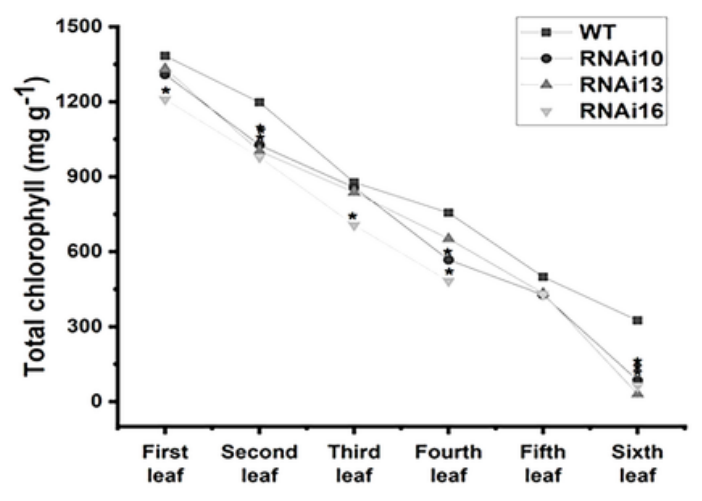

(b)

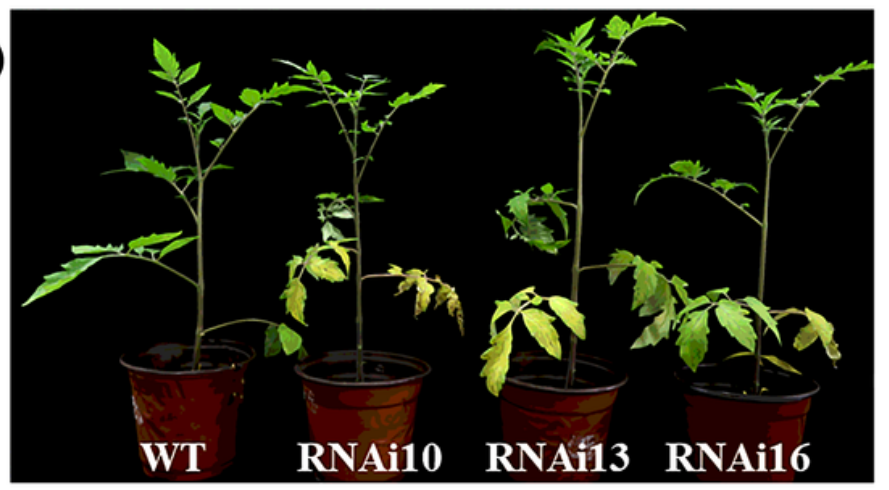

(d)

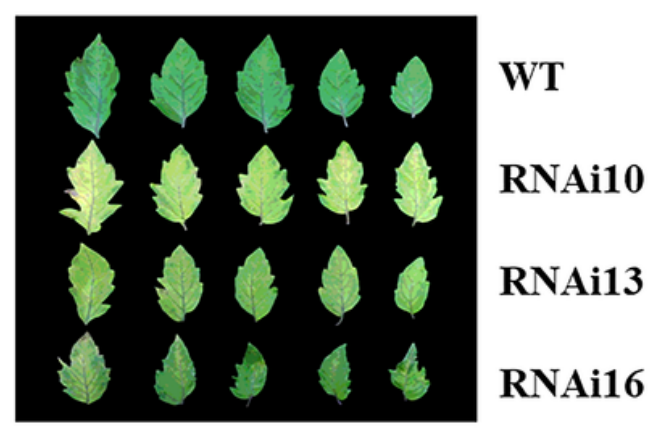

(f)

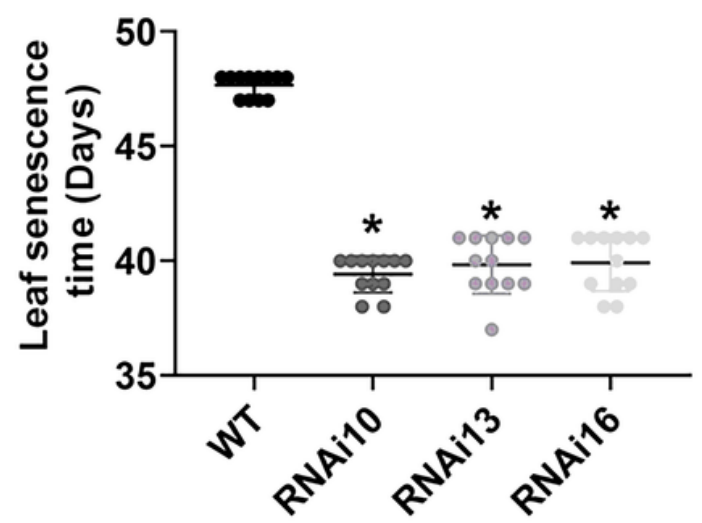

Figure 3

Silencing of SIERF.F5 causes premature senescence of tomato leaves (a) The expression level of SIERF.F5 in mature leaves of WT and SIERF.F5-RNAi lines. (b) The senescence phenotype of 10 weeks old WT, RNAi10, RNAi13, and RNAi16 lines. (c) The senescence phenotype of the fifth leaf of WT, RNAi10, RNAi13, and RNAi16 lines. (d) Isolation of leaves from 12-week-old WT and RNAi10, RNAi13, RNAi16 lines. (e) Chlorophyll content of each leaf in WT, RNAi10, RNAi13, and RNAi16 lines. (f) Leaf senescence time of WT, RNAi10, RNAi13 and RNAi16 lines. Three biological replicates were performed for each content determination. The data represent the mean from three replicates with three biological repeats. *, indicate $\mathrm{P}<0.05$, between the wild type and others by t-test. Error bars indicate SE. 

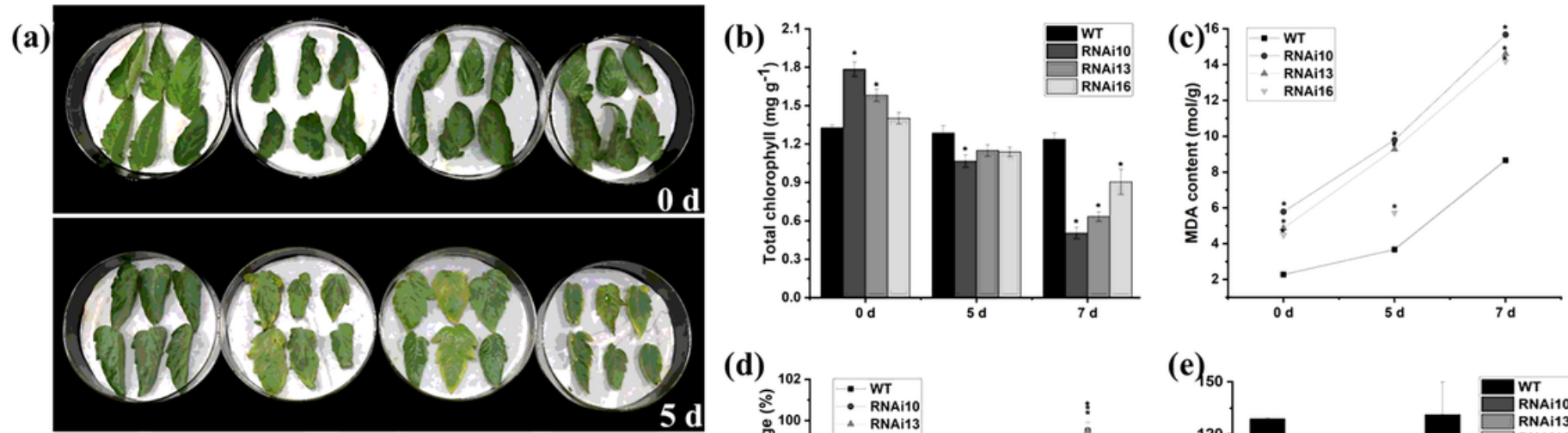

(d)
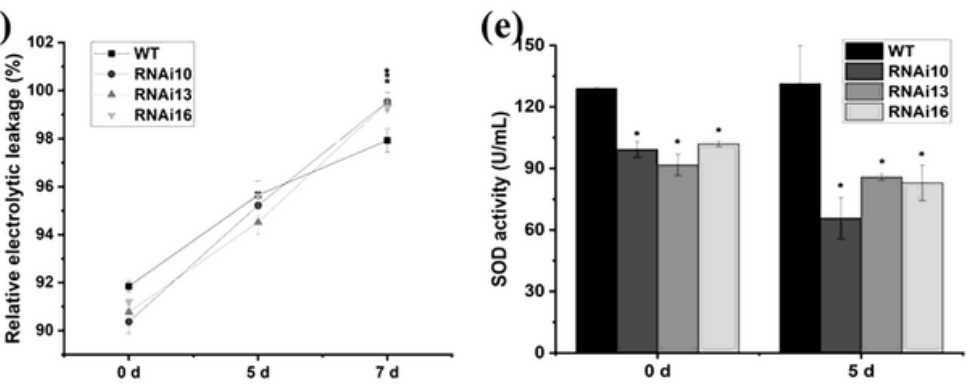

Figure 4

Silencing of SIERF.F5 promotes dark-induced leaf senescence (a) The leaves of the WT and SIERF.F5RNAi lines (RNAi10, RNAi13, RNAi16) were treated in the dark for 0 days, 5 days, and 7 days. (b) Leaf chlorophyll content of WT and RNAi10, RNAi13, RNAi16 lines at 0, 5, and 7 days in the dark. (c) The MDA (malonaldehyde) content of the leaves of the WT and RNAi10, RNAi13, RNAi6 lines at 0, 5, and 7 days of dark treatment. (d) Relative conductivity of leaves of WT and RNAi10, RNAi13, and RNAi16 lines in dark treatment for 0 days, 5 days, 7 days. (e) SOD activity in leaves of WT and RNAi10, RNAi13, and RNAi16 lines at 0 and 5 days after dark treatment. The data represent the mean from three replicates with three biological repeats. * ${ }^{*}$ indicate $\mathrm{P}<0.05$, between the wild type and others by t-test. Error bars indicate SE. 
(a)

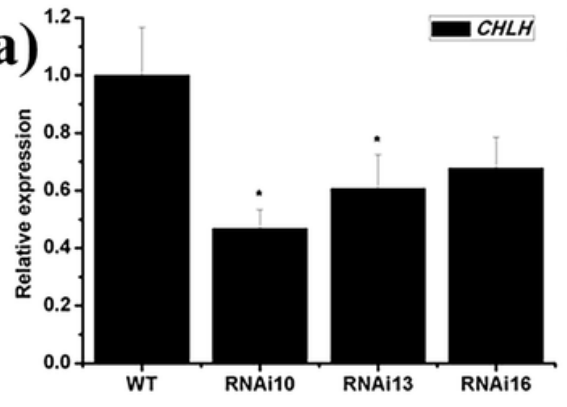

(d)

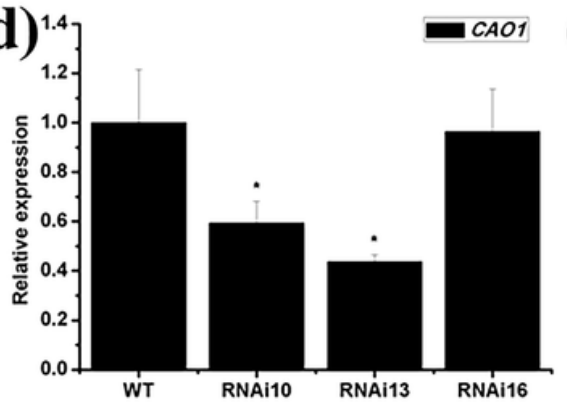

(g)

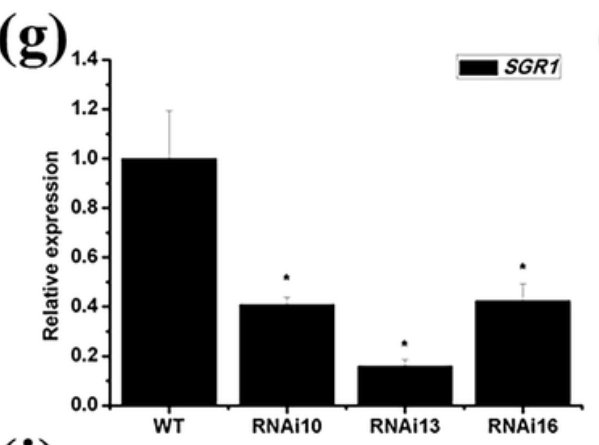

(j)

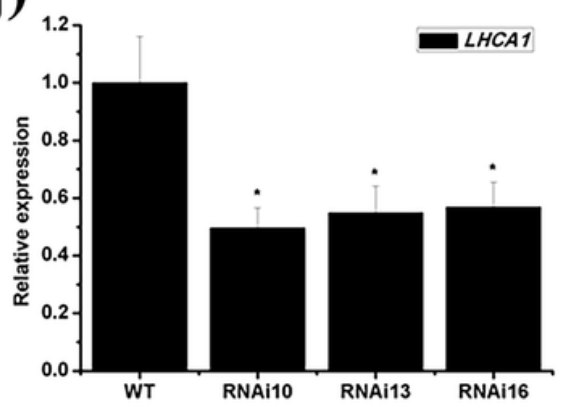

(b)

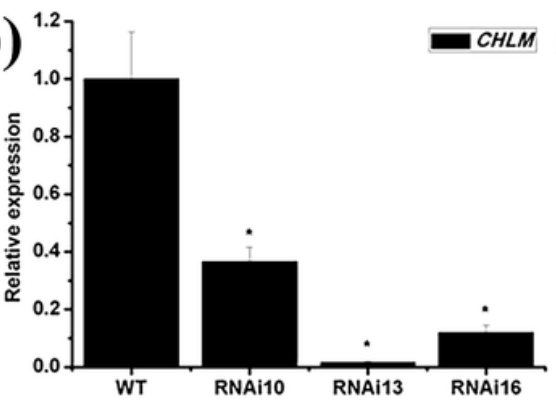

(e)

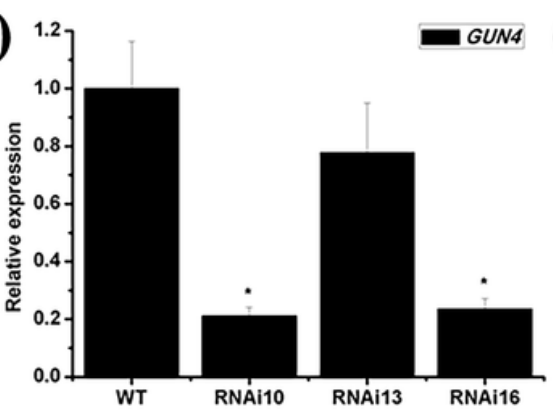

(h)

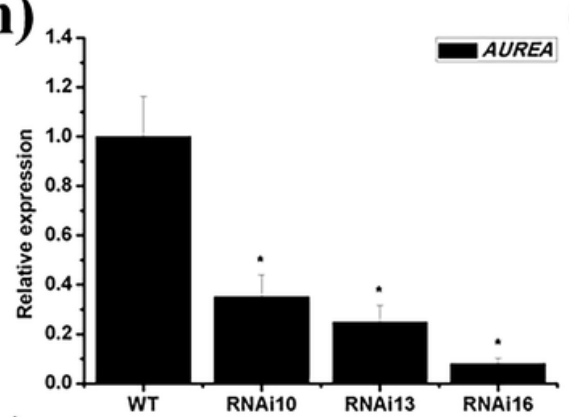

(k)

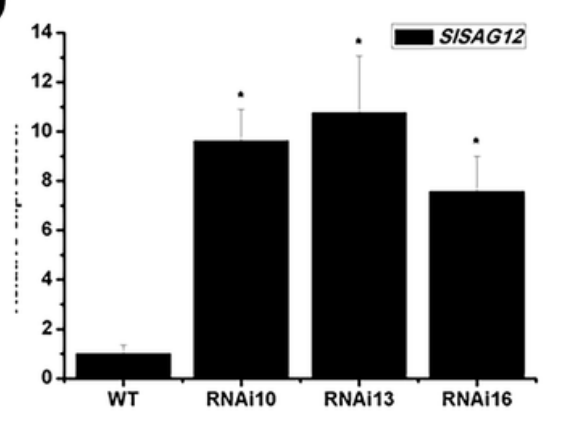

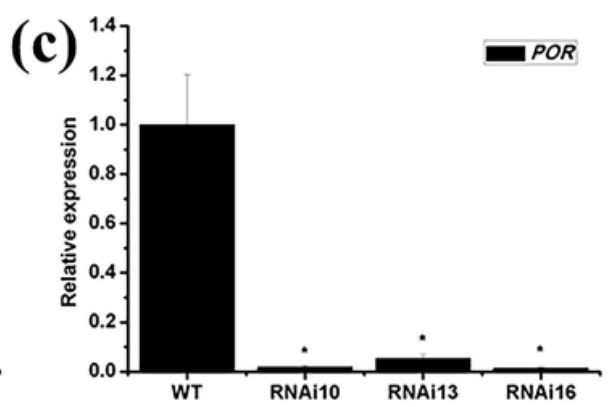

(f)

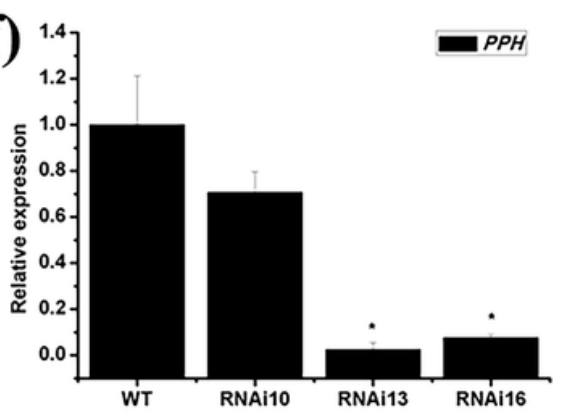

(i)

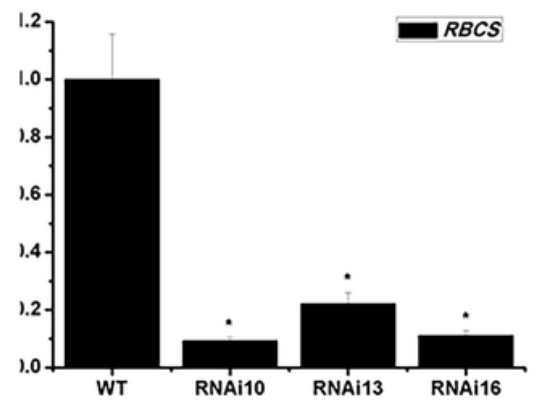

\section{Figure 5}

Silencing of SIERF.F5 altered the expression of genes involved chloroplast development, chlorophyll metabolism, and senescence markers (a-e) qRT-PCR analysis of CHLH, CHLM, POR, CA01, GUN4 (chloroplast development-related genes) expression levels in WT and RNAi10, RNAi13, RNAi16 lines. (f-i) qRT-PCR analysis of chloroplast metabolism related genes PPH, SGR1, RBCS, AUREA expression levels in WT and RNAi10, RNAi13, RNAi16 lines. (j) Quantitative RT-PCR analysis of the expression level of lightresponsive gene LHCA1 in WT and RNAi10, RNAi13, RNAi16 lines. (k) qRT-PCR analysis of the expression level of senescence marker gene SISAG12 in WT and RNAi10, RNAi13, RNAi16 lines. The data represent 
the mean from three replicates with three biological repeats. * , indicate $P<0.05$, between the wild type and others by t-test. Error bars indicate SE.

(a)

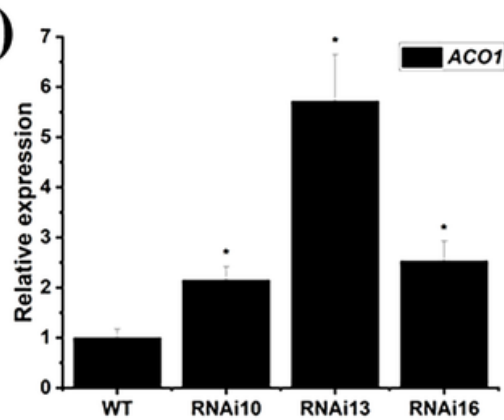

(d)

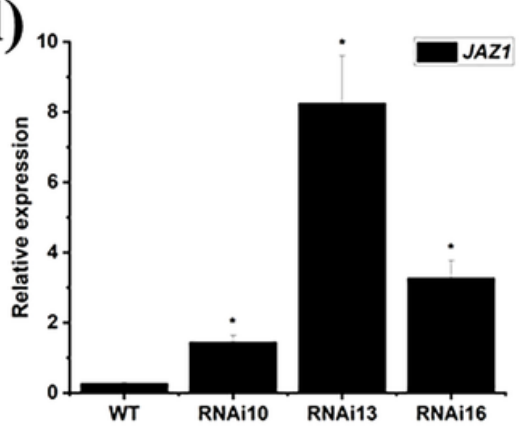

(g)

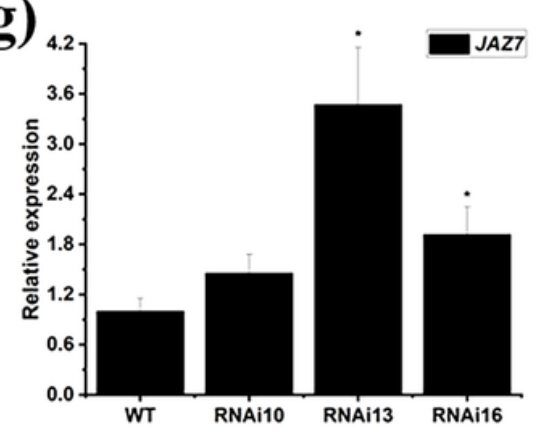

(j)

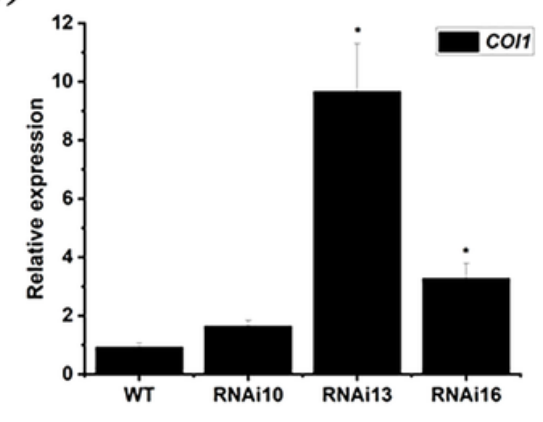

(b)

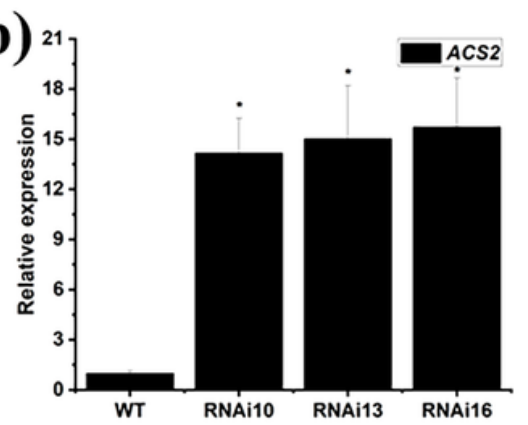

(e)

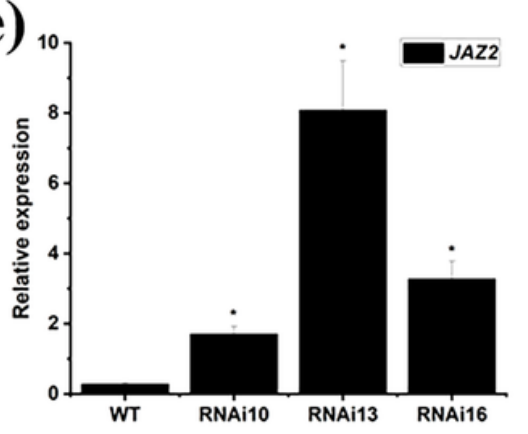

(h)

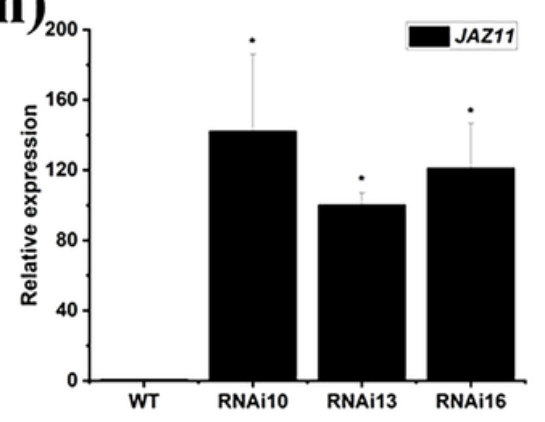

(c)

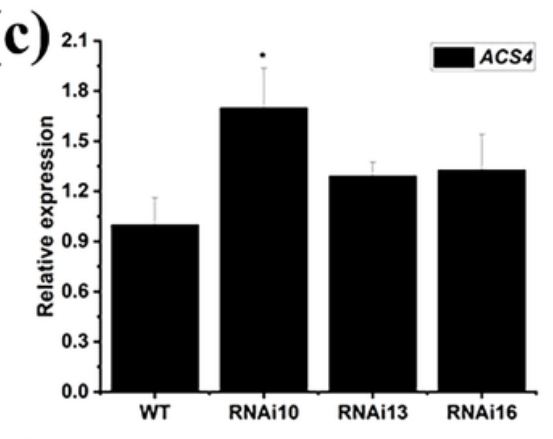

(f)

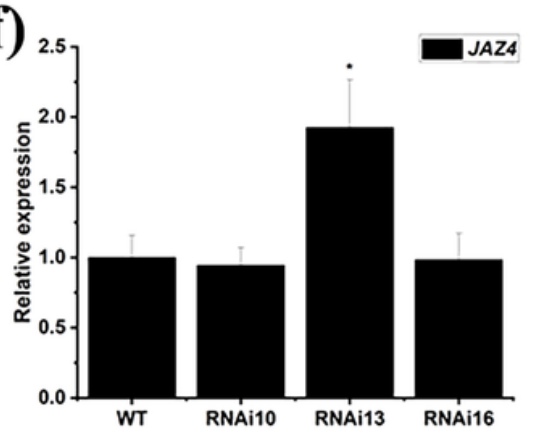

(i)

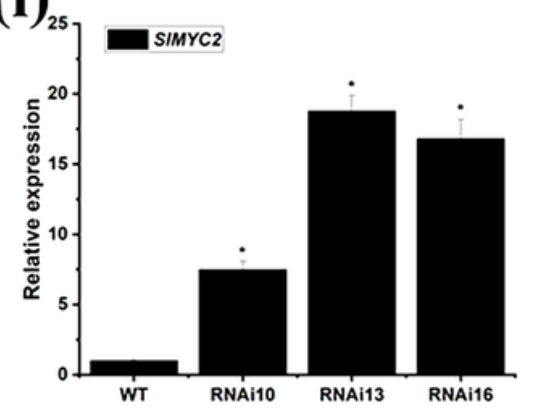

\section{Figure 6}

Silencing of SIERF.F5 affects the expression of ethylene, and jasmonic acid signaling pathway genes (a-

c) qRT-PCR analysis of the expression levels of ethylene biosynthetic pathway genes AC01, ACS2 and ACS4 in WT and RNAi10, RNAi13, RANi16 lines. (d-h) Quantitative RT-PCR analysis of jasmonic acid signal transduction pathway genes JAZ1, JAZ2, JAZ4, JAZ7, and JAZ11 expression levels in WT and RNAi10, RNAi13, RANi16 lines. (i-j) qRT-PCR analysis of the expression levels of jasmonic acid receptor 
downstream transcription factor MYC2 and jasmonic acid-dependent transcription factor COI1 in WT and RNAi10, RNAi13, RNAi16 lines. The data represent the mean from three replicates with three biological repeats. *, indicate $P<0.05$, between the wild type and others by t-test. Error bars indicate SE.

(a)

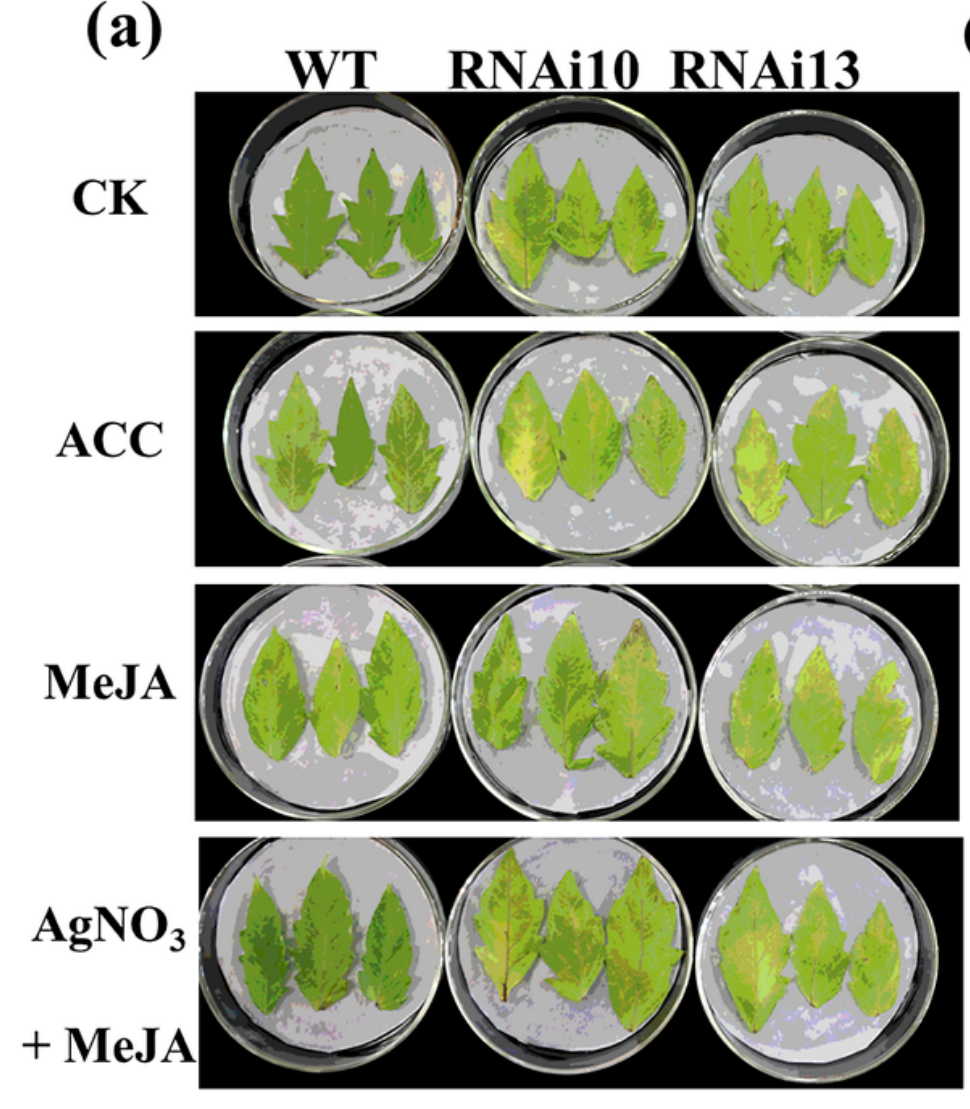

(b)

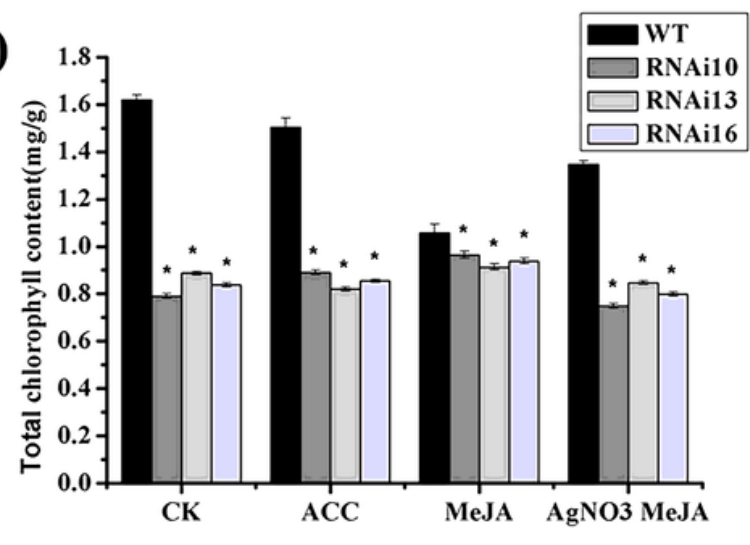

(c)

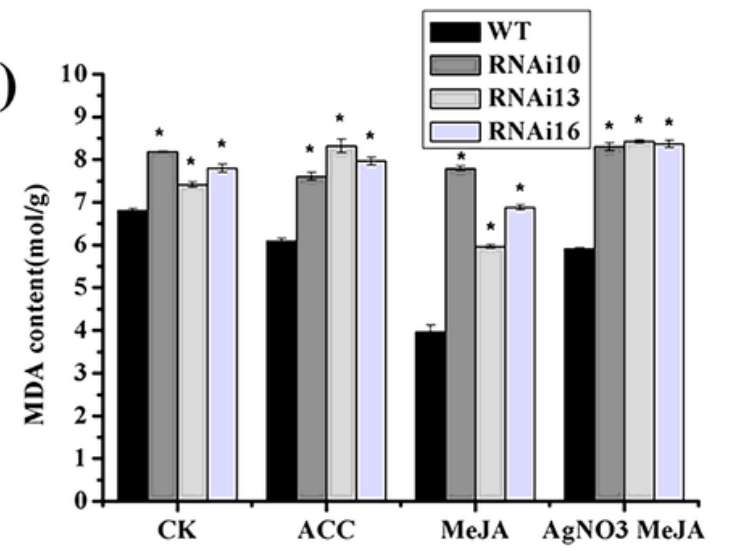

Figure 7

Silencing of SIERF.F5 promotes dark, ethylene and JA induced leaf senescence (a) Senescence phenotypes of isolated leaves of WT, RNAi10, RNAi13, RNAi16 lines treated with ACC, MeJA, and AgNO3+MeJA in the dark. Under dark conditions, the isolated leaves were treated with water (control), $100 \mu \mathrm{M}$ ACC, or $50 \mu \mathrm{M}$ MeJA for seven days. For AgNO3+MeJA treatment, the leaves were pretreated with $10 \mu \mathrm{M}$ AgNO3 for $1 \mathrm{~h}$, washed with water, and then treated with $50 \mu \mathrm{M}$ MeJA in the dark for seven $\mathrm{d}$. (b) The measurement of chlorophyll content comes from hormone-treated isolated leaves. (c) MDA content was measured from hormone-treated leaves. The data represent the mean from three replicates with three biological repeats. *, indicate $\mathrm{P}<0.05$, between the wild type and others by t-test. Error bars indicate SE. 
(a)

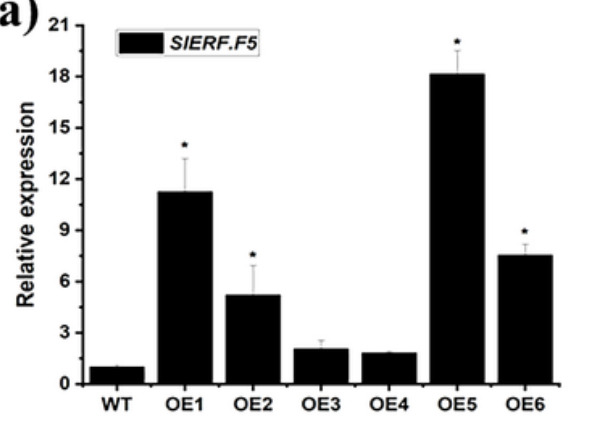

(c)

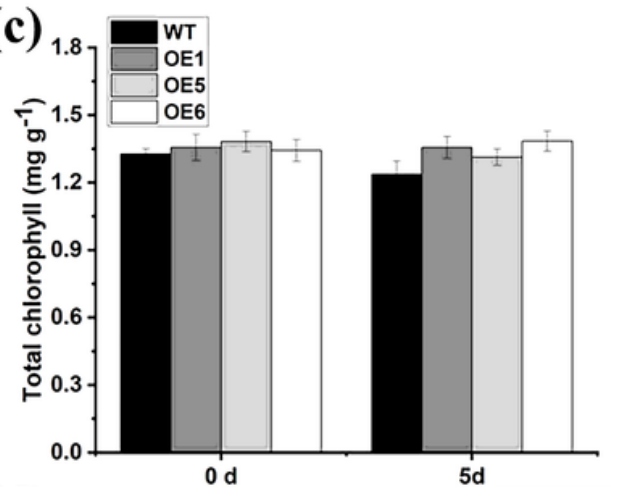

(d)

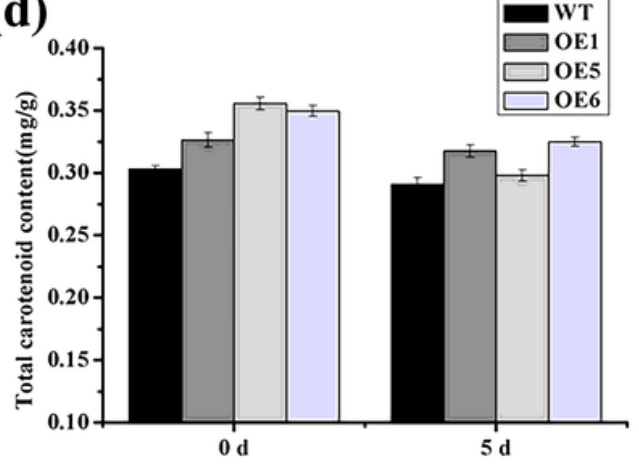

(b)
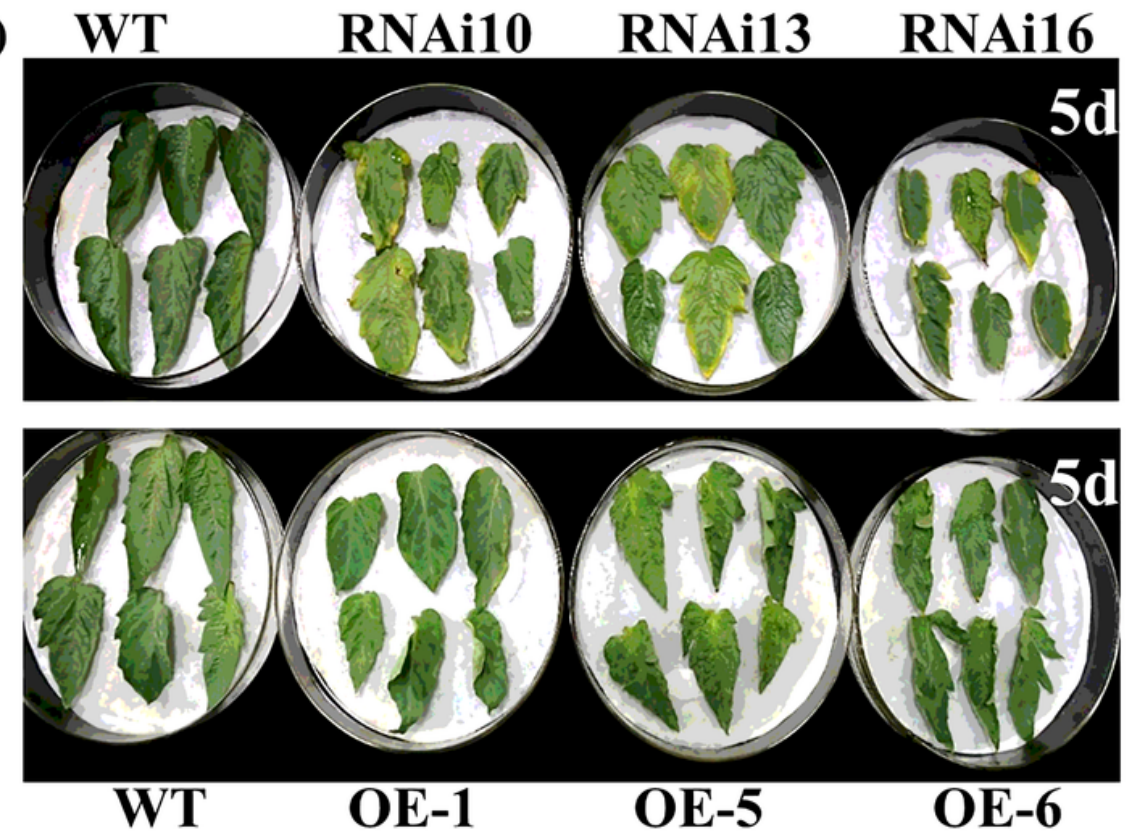

(e)

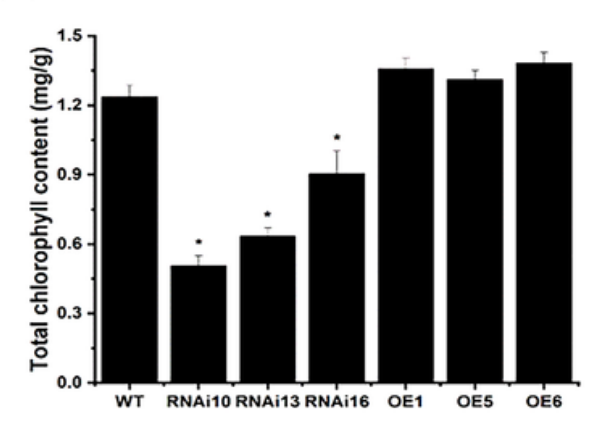

(f)

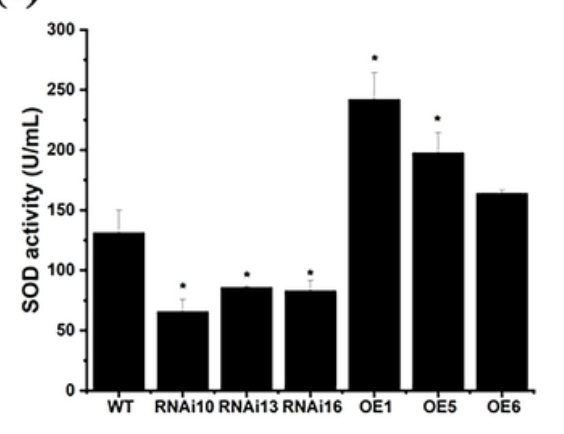

Figure 8

Overexpression of SIERF.F5 does not promote leaf senescence (a) The expression level of SIERF.F5 in mature leaves of WT and SIERF.F5-OE lines. (b) Dark-induced leaf senescence for five days in WT and RNAi10, RNAi13, RNAi16 lines and OE-1, OE-5, OE-6 lines. (c) Analysis of chlorophyll content in WT and OE-1, OE-5, OE-6 lines. (d) Analysis of carotenoid content in WT and OE-1, OE-5, OE-6 lines. (e) Analysis of chlorophyll content in WT and RNAi10, RNAi13, RNAi16 lines and OE-1, OE-5, OE-6 lines. (f) Analysis of SOD activity in WT and RNAi10, RNAi13, RNAi16 lines and OE-1, OE-5, OE-6 lines. The data represent the mean from three replicates with three biological repeats. *, indicate $\mathrm{P}<0.05$, between the wild type and others by t-test. Error bars indicate SE. 
$B D$

BD-SIERF.F5

pGBKT7-Lam pGADT7-T(-)

pGBKT7-53 pGADT7-T(+)

BD-SIERF.F5 AD

BD-SIERF.F5

$A D$
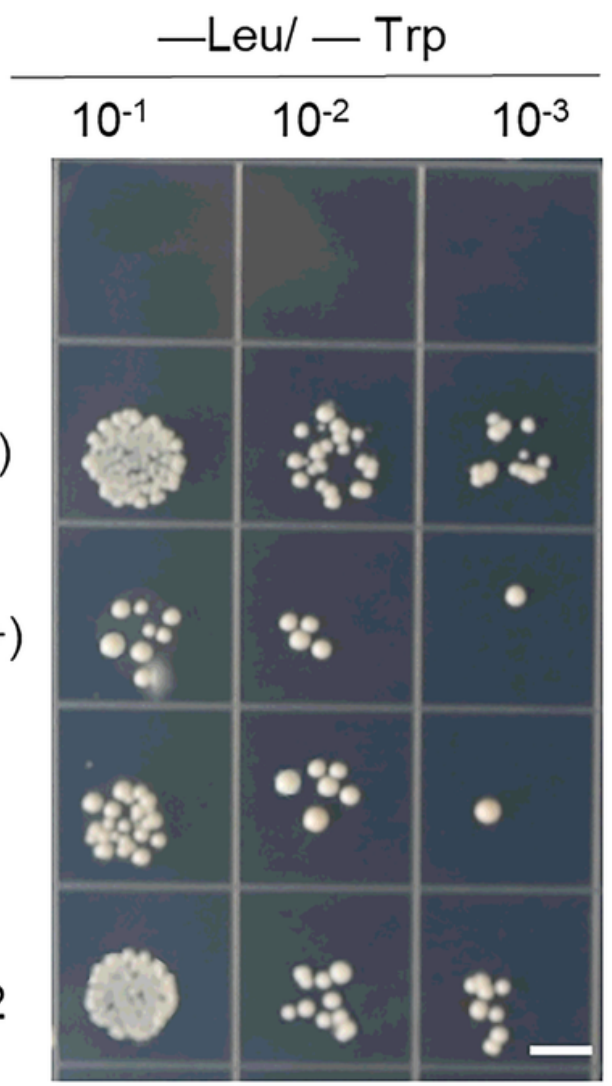

- Leu/_Trp/

\begin{tabular}{cc}
\multicolumn{3}{c}{-Ade/—His } \\
\hline $10^{-1} \quad 10^{-2} \quad 10^{-3}$
\end{tabular}

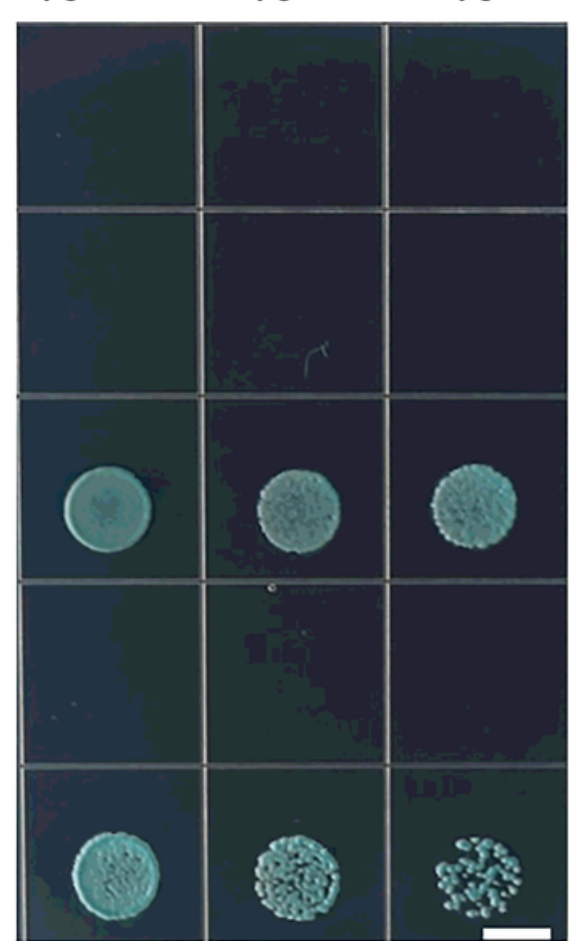

\section{Figure 9}

SIERF.F5 Physically Interacts with SIMYC2 Yeast two-hybrid experiment indicated that SIERF.F5 interacted with SIMYC2. Co-transformation of pGADT7-T and pGBKT7-53 as a positive control; co-transformation of pGADT7-T and pGBKT7-Lam as a negative control; single-transformation of BD-SIERF.F5 and cotransformation with AD to verify self-activation; co-transformation of BD-SIERF.F5 and AD-SIMYC2 as an experimental group. The whitewire frame represents $1 \mathrm{~cm}$. 


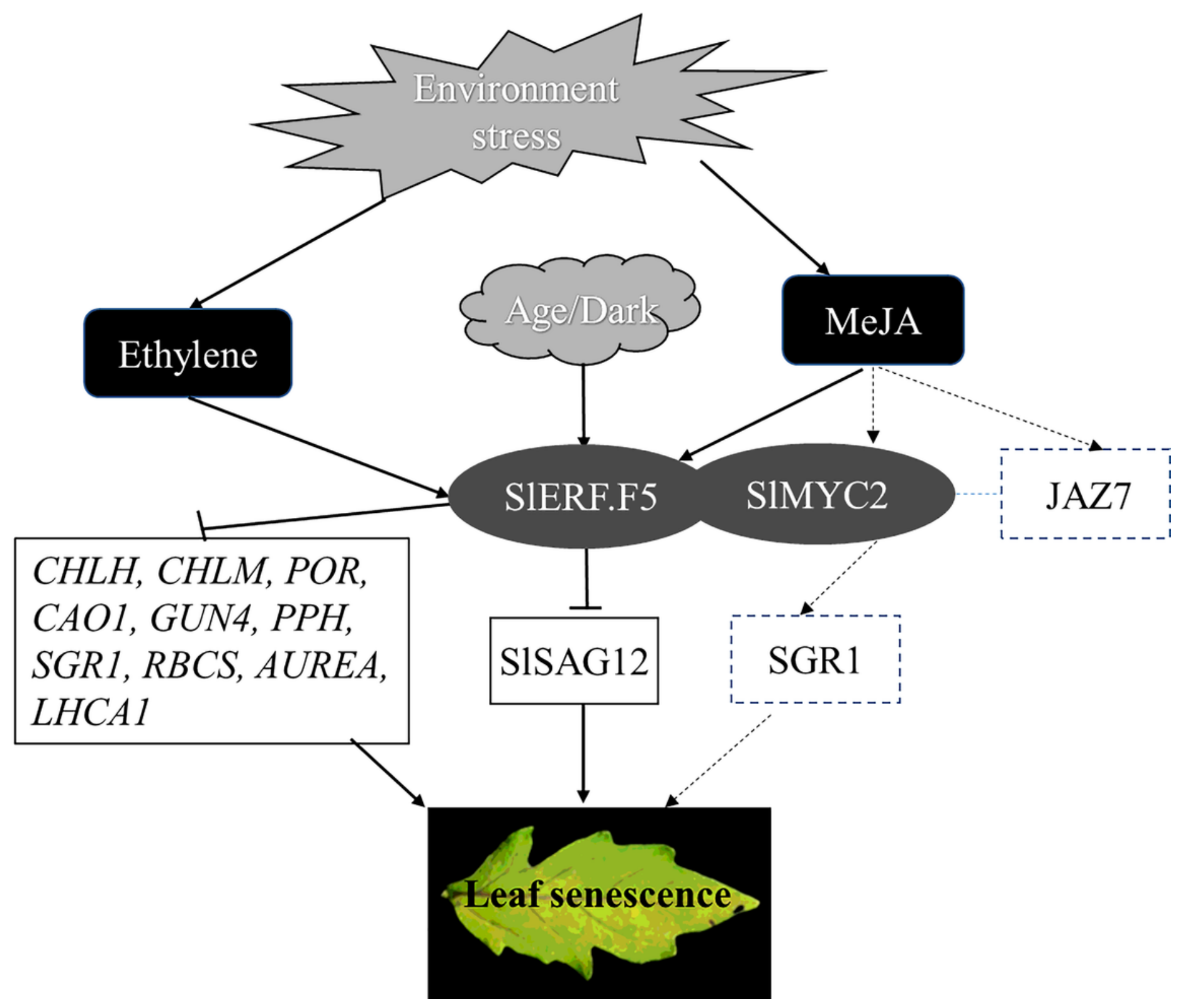

Figure 10

A proposed model illustrates the cascade regulation of SIERF.F5-SIMYC2 in leaf senescence

\section{Supplementary Files}

This is a list of supplementary files associated with this preprint. Click to download.

- Fig.S1.tif

- Fig.S2.tif

- supplementary.docx 